\title{
Identification of N-nitrosamines and nitrogenous heterocyclic byproducts during chloramination of aromatic secondary amine precursors
}

Xueke Liu ${ }^{1}$, Yongfeng $\operatorname{Lin}^{1,2}$, Ting Ruan ${ }^{1,2 *}$ and Guibin Jiang ${ }^{1,2}$

${ }^{1}$ State Key Laboratory of Environmental Chemistry and Ecotoxicology, Research Center for Eco-Environmental Sciences, Chinese Academy of Sciences, Beijing 100085, China

${ }^{2}$ University of Chinese Academy of Sciences, Beijing 100049, China

*Corresponding author

Dr. Ting Ruan

Research Center for Eco-Environmental Sciences,

Chinese Academy of Sciences

Tel: 8610-6284-9334

Fax: 8610-6284-9179

E-mail: tingruan@rcees.ac.cn

Number of pages: 38

Number of Tables: 7

Number of Figures: 16 


\title{
CONTENTS:
}

\section{Appendix S1. Chemicals and Reagents}

\section{Appendix S2. Solid Phase Extraction}

\author{
Appendix S3. Instrumental Analysis
}

Appendix S4. Quality Assurance and Quality Control (QA/QC)

Appendix S5. Selection of Quenching Agents for N-chlorinated Products

Appendix S6. The Chloramination Experiment of Phenazine

\section{References}

Table S1. Nitrosamines in carcinogen lists by International Agency for Research on Cancer, and nitrosamine DBPs in EPA's Drinking Water Contaminant Candidate List 4 (CCL 4) and Unregulated Contaminant Monitoring Rule 2 (UCMR 2).

Table S2. Detailed information on CAS number, chemical name, annual production, molecular structure and formula of registered diaromatic amines in USA and EU.

Table S3. Detailed information on chemical name, annual production, molecular structure and formula of registered $\mathrm{N}$-alkyl aromatic amines in USA and EU.

Table S4. Detailed information on CAS number, chemical name, abbreviated name, formula, structure, and supplier of the target secondary amine precursors and nitrosamine analogues used in this study.

Table S5. ${ }^{14} \mathrm{~N} /{ }^{15} \mathrm{~N}$-DBP pairs obtained from the N-DBPs screening workflow after the reaction between DPA $(5 \mu \mathrm{M})$ and chloramines $\left(0.5 \mathrm{mM}\right.$ with $\left.{ }^{14} \mathrm{~N} /{ }^{15} \mathrm{~N}=1.0\right)$ at $\mathrm{pH}=7$ for 12-hour treatment.

Table S6. Detailed information on characteristic $\mathrm{MS}^{2}$ fragment ions, existence of $\mathrm{N}-\mathrm{Cl}$ bond, and structure elucidation confidence level of the identified N-DBPs in chloramination of DPA.

Table S7. Effect of DOM (3.0 mg/L as C) and precursor concentrations on formation of Group I-IV N-DBPs after $0.5 \mathrm{mM}$ chloramine disinfection at $\mathrm{pH} 7$ for 12 hours. 
Figure S1. Scheme of N-DBP screening workflow. $(A)$ Formation of NDMA through chlorinated unsymmetrical dimethylhydrazine intermediate in chloramination of DMA. (B) Theoretical formation of ${ }^{14} \mathrm{~N}-\mathrm{NDPhA}$ and ${ }^{15} \mathrm{~N}-\mathrm{NDPhA}$ in chloramination of DPA with equivalent molar amounts of ${ }^{14} \mathrm{~N}$-chloramines and ${ }^{15} \mathrm{~N}$-chloramines. (C) Theoretical chromatographic retention behaviors and ions signals of ${ }^{14} \mathrm{~N}-\mathrm{DBPs}$ and ${ }^{15} \mathrm{~N}$ DBPs. That is, ${ }^{14} \mathrm{~N}$-DBPs and ${ }^{15} \mathrm{~N}$-DBPs have the same retention time, and the corresponding ions have the same intensity as well as a mass difference of $0.99704( \pm$ 0.5 ppm Error Threshold).

Figure S2. N-DBP screening workflow, and the method performance for identification of potential N-DBPs in chloramination of DPA. $(A)$ Screening workflow for feature detection, peak pair filtering, and structure confirmation of N-DBPs. $(B)$ Distribution of the number of N-DBPs in which the mass differences of ${ }^{14} \mathrm{~N} /{ }^{15} \mathrm{~N}-D B P s$ were within the range of $-0.5-0.5 \mathrm{ppm}$ (Error Threshold) compared with the theoretical value of 0.99704. (C) Distribution of the number of N-DBPs with intensity ratios of ${ }^{14} \mathrm{~N} /{ }^{15} \mathrm{~N}$ DBPs in the range of $1.0 \pm 0.3$. $(D)$ The same retention behaviors and fragmentation patterns of ${ }^{14} \mathrm{~N} /{ }^{15} \mathrm{~N}-\mathrm{NDPhA}$ (blue and red) produced in chloramination and NDPhA standard (black), respectively. Data was from the reaction between DPA and chloramines with molar ratios of chloramines/precursor (100) at $\mathrm{pH} 7$ after $12 \mathrm{~h}$.

Figure S3. Recoveries of the targeted standards of diaromatic and N-alkyl aromatic secondary amine precursors, and N-nitrosamines (10 $\mathrm{nM}$ of spiked concentration for each analyte in $10 \mathrm{mM}$ phosphate buffer solution) by using the solid phase extraction method.

Figure S4. Stability of instrumental responses of the N-DBP analogues formed in the chloramination reactions by measuring quality control (QC) sample throughout batches of sample. The QC was prepared by mixing $10 \mu \mathrm{L}$ each sample in the experiments on chloramination of DPA at distinct disinfectant doses, $\mathrm{pH}$ values and reaction time.

Figure S5. Ratios of ${ }^{15} \mathrm{~N}-\mathrm{DBPs} /{ }^{14} \mathrm{~N}$-DBPs peak areas produced in chloramination of DPA with different reaction conditions, including $\mathrm{pH}$ values $(A)$, chloramines doses $(B)$ and reaction time $(C)$. Ratios of all N-DBPs were within a reasonable range of 0.7 to 1.3, except for those of NDPhA and HO-NDPhA less than 0.7 under a few conditions. The ${ }^{15} \mathrm{~N}-\mathrm{DBP} /{ }^{14} \mathrm{~N}-\mathrm{DBP}$ peak area ratios of $\mathrm{NDPhA}$ and $\mathrm{HO}-\mathrm{NDPhA}$ gradually approached 1.0 with the increase of reaction time shown in the dynamics diagram $(D)$.

Figure S6. The same retention behaviors and fragmentation patterns of ${ }^{14} \mathrm{~N} /{ }^{15} \mathrm{~N}$-ions were shown in monoisotopic mass (MIM) chromatograms and $\mathrm{MS}^{2}$ spectra of $\mathrm{HO}-$ NDPhA and Cl-NDPhA produced in chloramination of DPA, respectively. (A) Neutral losses of $\mathrm{OH}$ and $\mathrm{NO}$ in $\mathrm{MS}^{2}$ spectra of ${ }^{14} \mathrm{~N} /{ }^{15} \mathrm{~N}-\mathrm{HO}-\mathrm{NDPhA}$. (B) Neutral losses of $\mathrm{Cl}$ and NO in $\mathrm{MS}^{2}$ spectra of ${ }^{14} \mathrm{~N} /{ }^{15} \mathrm{~N}-\mathrm{Cl}-\mathrm{NDPhA}$.

Figure S7. Proposed formation pathway of Cl-DiH-Phe (A), Phe (B) and Cyc-DiDPA 
(C) from the reaction of DPA with dichloramine. (A) A carbanion resonance structure of DPA successively reacted with dichloramine to form instantaneous intermediate 5,10-dihydro-phenazine (DiH-Phe), and then DiH-Phe was rapidly chlorinated by chloramines to form Cl-DiH-Phe. (B) By elimination reaction of intramolecular resonance to lose the $\mathrm{N}-\mathrm{Cl}$ bond, $\mathrm{Cl}-\mathrm{DiH}-\mathrm{Phe}$ could further produce Phe. (C) By attacking $\mathrm{N}-\mathrm{Cl}$ with the carbanion in a resonance structure to lose a $\mathrm{HCl}, \mathrm{Cl}$-DiDPA with $\mathrm{N}-\mathrm{Cl}$ could further form Cyc-DiDPA.

Figure S8. After quenching by $2 \mathrm{mM}$ ascorbic acid (green), ${ }^{14} \mathrm{~N} /{ }^{15} \mathrm{~N}-\mathrm{Cl}-\mathrm{DiH}-\mathrm{Phe}$ (blue and red) disappeared as shown in the monoisotopic mass (MIM) chromatograms, suggesting existence of the $\mathrm{N}-\mathrm{Cl}$ bond. The reaction conditions were with chloramines/DPA molar ratios of 100/1 at $\mathrm{pH} 7$ for $12 \mathrm{~h}$.

Figure S9. The same retention behaviors and fragmentation patterns of ${ }^{14} \mathrm{~N} /{ }^{15} \mathrm{~N}-\mathrm{Phe}$ (blue and red) produced in chloramination and Phe standard (black) shown in monoisotopic mass (MIM) chromatograms and $\mathrm{MS}^{2}$ spectra, respectively. A pair of fragment ions $\left(\left[\mathrm{C}_{11} \mathrm{H}_{8}{ }^{14} \mathrm{~N}\right]^{+}\right.$and $\left.\left[\mathrm{C}_{11} \mathrm{H}_{8}{ }^{15} \mathrm{~N}\right]^{+}\right)$with similar intensity were shown in $\mathrm{MS}^{2}$ spectrum of ${ }^{15} \mathrm{~N}$-Phe, indicating ${ }^{14} \mathrm{~N}$ (originally from the DPA molecule) and ${ }^{15} \mathrm{~N}$ atoms (from chloramines) were in the enantiomeric position in ${ }^{15} \mathrm{~N}-\mathrm{Phe}$.

Figure S10. Concentrations of monochloramine and dichloramine in the $0.5 \mathrm{mM}$ solutions of chloramines $\left(\right.$ as $\left.\mathrm{Cl}_{2}\right)$ at $\mathrm{pH} 4-10$.

Figure S11. Effect of $\mathrm{pH}$ on formation of the four groups of N-DBPs and hydroxylated/chlorinated products. Maximum molar yields of all chlorinated products were observed at $\mathrm{pH} \leqslant 7$. Except for HO-Cl-DiH-Phe, maximum formation yields of hydroxylated products were found at $\mathrm{pH} \geqslant 7$.

Figure S12. Effect of chloramine doses on formation of the four groups of N-DBPs and hydroxylated/chlorinated products, in which maximum molar yields of hydroxylated products were found with molar ratios of chloramines/DPA at $10-100$, and dependences of chlorinated DBPs on disinfectants doses were noticed.

Figure S13. Main reaction conditions for preferred formation of the four groups of NDBPs from DPA.

Figure S14. Increased retention of monochlorinated cyclized quinonediimines with growth of alkyl chain lengths was shown in the monoisotopic mass (MIM) chromatograms. Fragment ions $\left[\mathrm{C}_{8} \mathrm{H}_{7}{ }^{14} \mathrm{~N}_{2} \mathrm{Cl}\right]^{+}$and $\left[\mathrm{C}_{8} \mathrm{H}_{7}{ }^{14} \mathrm{~N}^{15} \mathrm{NCl}\right]^{+}$with the maximum abundance as shown in $\mathrm{MS}^{2}$ spectra of ${ }^{14} \mathrm{~N}$ and ${ }^{15} \mathrm{~N}$ peaks were cyclized molecular skeleton. Monochlorinated cyclized quinonediimines were produced in chloramination of N-ethylaniline (A), N-propylaniline (B), N-butylaniline (C), and Ncyclohexylaniline (D). 
Figure S15. Molar yields of N-nitrosamines from different secondary amine precursors with chloramines/precursor molar ratios of 100/1 at pH 7 after $12 \mathrm{~h}$ treatment. Higher molar yields from $\mathrm{N}$-alkyl aromatic and diaromatic amines were observed compared with those from dialkyl amines.

Figure S16. Main fragmentation patterns of different N-nitrosoamines in $\mathrm{MS}^{2}$ spectra acquired by HRMS at $30 \% \mathrm{HCD}$ energy. Diagnostic loss of NO was observed in $\mathrm{MS}^{2}$ spectra of $\mathrm{N}$-nitroso diaromatic amines, but loss of alkyl moieties was specific for $\mathrm{N}$ nitroso dialkyl amines. Losses of both the NO and alkyl moieties appeared in $\mathrm{MS}^{2}$ spectra of N-nitroso alkyl aromatic amines, which were related to the length of alkyl moieties. It should be noted that standards of $\mathrm{N}$-nitrosopropylaniline and $\mathrm{N}$ nitrosobutylaniline were not commercially available, and the $\mathrm{MS}^{2}$ spectra were obtained from the chloramine-treated samples of corresponding amine precursors. 


\section{Appendix S1. Chemicals and Reagents}

Authentic standards of diaromatic, dialkyl and N-alkyl aromatic DPA analogues, and nine nitrosamine chemicals were obtained commercially. Detailed information on CAS number, chemical name, abbreviated name, formula and supplier of chemicals was shown in Table S4. The purities of all standards were no less than $95 \%$ unless otherwise stated. Ammonium chloride $\left({ }^{14} \mathrm{NH}_{4} \mathrm{Cl}\right)$ and sodium hypochlorite solution $(4.00-4.99 \%$ available chlorine) were purchased from Sigma-Aldrich (St. Louis, MO). Isotopelabeled ammonium chloride $\left({ }^{15} \mathrm{NH}_{4} \mathrm{Cl}\right)$ was acquired from Cambridge Isotope Laboratories (Andover, USA). Suwannee River fulvic acid (SRFA, 2S101F) was obtained from the International Humic Substances Society. HPLC-grade methanol and dichloromethane were obtained from Fisher Chemical (Fair Lawn, NJ). Ultrapure water $(18.3 \mathrm{M} \Omega \cdot \mathrm{cm})$ was generated by a Milli-Q system (Millipore, Billerica, MA).

\section{Appendix S2. Solid Phase Extraction}

Briefly, $40 \mathrm{~mL}$ of aqueous samples were adjusted to $\mathrm{pH}=\sim 8.0$ by sodium hydroxide solution $(1 \mathrm{M})$ or hydrochloric acid $(1 \mathrm{M})$, which were then loaded into the HLB cartridges. Before extraction, the cartridges were rinsed by $10 \mathrm{~mL}$ of dichloromethane, $10 \mathrm{~mL}$ of methanol and $10 \mathrm{~mL}$ of ultrapure water, successively. After sample loading at a flow rate of $\sim 6 \mathrm{~mL} / \mathrm{min}$, the cartridges were desalted by $5 \mathrm{~mL}$ of ultrapure water, and then eluted by $10 \mathrm{~mL}$ of dichloromethane. The eluates were concentrated under a gentle nitrogen stream, and then reconstituted into $200 \mu \mathrm{L}$ of methanol for further instrumental analysis.

\section{Appendix S3. Instrumental Analysis}

An Orbitrap Fusion mass spectrometer coupled with Ultimate 3000 ultrahigh performance liquid chromatography (UPLC-Orbitrap HRMS, Thermo Fisher Scientific Inc., Waltham, MA) was applied for chemical feature detection and molecular structure 
characterization. An ACQUITY BHE C18 column (Waters, $1.7 \mu \mathrm{m}, 2.1 \times 100 \mathrm{~mm}$ ) was used for analyte separation. Flow rate was set as $0.2 \mathrm{~mL} / \mathrm{min}$, column temperature was set at $35^{\circ} \mathrm{C}$, and injection volume was $5 \mu \mathrm{L}$. The mobile phase consisted of methanol (A) and water (B), with each phase containing $1 \mathrm{mM}$ ammonium acetate. A gradient program was performed as follow: hold $40 \%$ of A for 5 min, linearly increased to $90 \%$ of $\mathrm{A}$ in $1 \mathrm{~min}$, and hold $90 \%$ of A for another $14 \mathrm{~min}$. Finally, the column was rebalanced by holding $40 \%$ of A for 9 min.

Positive electrospray ionization (ESI) was utilized to acquire mass spectral information. Spray voltage was set as $3500 \mathrm{~V}$, and vaporizer temperature was $200{ }^{\circ} \mathrm{C}$. The pressures of sheath gas and aux gas were 35 and 15 Arb, respectively. The full scan mass spectra $\left(\mathrm{MS}^{1}\right)$ were obtained with the resolution of $120,000 \mathrm{fwhm}$ (full width at half-maximum at $\mathrm{m} / \mathrm{z} 200$ ), and the scan range of $\mathrm{m} / \mathrm{z}=50-1000 . \mathrm{MS}^{2}$ Spectra were acquired at the higher-energy collisional dissociation (HCD) mode. Collision energies were set in the range of $30-70 \%$ at a resolution of 60,000 (fwhm at $\mathrm{m} / \mathrm{z} 200)$, and the $\mathrm{MS}^{1}$ precursors were isolated by quadrupole with window of $\mathrm{m} / \mathrm{z}=1$.

The chloramines species $\left(0.5 \mathrm{mM}\right.$ as $\left.\mathrm{Cl}_{2}\right)$ were measured at different $\mathrm{pH}$ values $(4,5$, $6,7,8,9$ and 10 ) by the previous spectrophotometry method. ${ }^{\mathrm{S} 1}$ The molar absorption coefficients of monochloramine were 445 and $14 \mathrm{M} \mathrm{cm}^{-1}$ at 245 and $295 \mathrm{~nm}$, respectively. Molar absorption coefficients of dichloramine were 208 and $267 \mathrm{M} \mathrm{cm}^{-1}$ at 245 and $295 \mathrm{~nm}$, respectively.

\section{Appendix S4. Quality Assurance and Quality Control (QA/QC)}

Three diaromatic, five $\mathrm{N}$-alkyl aromatic DPA analogues and nine nitrosamines (10 nM) were spiked in $10 \mathrm{mM}$ phosphate buffer solution at $\mathrm{pH}=7$ for method performance evaluation (Figure S3). Quantification of the analytes was based on an external calibration curve by using the commercial native standards. The recoveries of analytes in the SPE pretreatment process were in the range of $23 \pm 5 \%$ (NMeA) to $94 \pm 8 \%$ 
(diAMS), among which the recoveries of DPA and NDPhA were $82 \pm 5 \%$ and $92 \pm$ $7 \%$, respectively. All data of the target analytes reported in the manuscript were not corrected for recoveries. Method quantification limits, based on a signal/noise ratio of ten, were $0.9 \mathrm{pM}$ (DPA, abbreviation shown in Table S4), $0.2 \mathrm{pM}$ (Phe), $0.7 \mathrm{pM}$ (NDPhA), 0.9 pM (NDEA), 0.5 pM (NDPA), 0.02 pM (NDBA), 0.2 pM (NPyr), 1.3 pM (NMor), $0.02 \mathrm{pM}$ (NDChA), $0.8 \mathrm{pM}$ (NNMeA) and $0.5 \mathrm{pM}$ (NNEtA), respectively. Quality control samples (QC, $\mathrm{n}=6$ ) were prepared by mixing $10 \mu \mathrm{L}$ of extracts from each sample after DPA chloramination treatments, which were injected in each batch of nine samples to monitor instrumental stability. As shown in Figure S4, instrumental responses (IRs) of DPA and corresponding ${ }^{14} \mathrm{~N} /{ }^{15} \mathrm{~N}-\mathrm{DBP}$ s remained in the range of 90 $-103 \%$ with relative standard deviations of $1.4-12 \%$. Interferent peak pairs, which were monitored in disinfection-control samples, accounted for $<10 \%$ of those found in experimental samples, and were thus excluded for further analysis. In the chloraminetreated DPA sample directly injected into instrument, the molar yields of NDPhAs, Phes, DiH-Phes and DiDPAs were semi-quantified as $11 \%, 18 \%, 58 \%$ and $3 \%$, respectively. After the sample was concentrated by SPE, the molar yields of NDPhAs, Phes, DiHPhes and DiDPAs were $11 \%, 20 \%, 55 \%$ and $3 \%$, respectively, which were not significantly different from those in the directly-injected samples.

Performance of the N-DBPs screening workflow was evaluated by using full-scan $\mathrm{MS}^{1}$ spectra information generated from the feature detection process. After reaction between DPA and isotopically-labeled chloramines $\left({ }^{15} \mathrm{~N} /{ }^{14} \mathrm{~N}=1.0\right.$, Table S5), 31 ${ }^{14} \mathrm{~N} /{ }^{15} \mathrm{~N}$-DBP pairs were discerned. As shown in Figure S2B, $71 \%$ of ${ }^{14} \mathrm{~N} /{ }^{15} \mathrm{~N}-\mathrm{DBP}$ pairs had accurate mass differences with errors less than $0.1 \mathrm{ppm}$, and $97 \%$ ones were within errors $<0.3 \mathrm{ppm}$. The intensity ratios of $90 \%{ }^{14} \mathrm{~N} /{ }^{15} \mathrm{~N}-\mathrm{DBP}$ peaks were in the range of $0.9-1.1$, which were close to the theoretical value of 1.0 (Figure S2C). A representative N-DBP (i.e. NDPhA) was identified by a 0.99703 mass difference of ${ }^{15} \mathrm{~N}-\mathrm{NDPhA}$ and ${ }^{14} \mathrm{~N}-\mathrm{NDPhA}$, which had only an $-0.07 \mathrm{ppm}$ error compared with the theoretical value, and had an intensity ratio of 1.01 in regard to ${ }^{15} \mathrm{~N}-\mathrm{NDPhA} /{ }^{14} \mathrm{~N}$ $\mathrm{NDPhA}$. Besides, the same chromatographic retention behaviors and fragmentation 
patterns of ${ }^{14} \mathrm{~N} /{ }^{15} \mathrm{~N}-\mathrm{NDPhA}$ in the $\mathrm{MS}^{2}$ spectra were observed. The neutral loss of ${ }^{15} \mathrm{NO}$ in the $\mathrm{MS}^{2}$ spectrum of ${ }^{15} \mathrm{~N}-\mathrm{NDPhA}$ suggested that nitrogen in nitroso moiety was derived from chloramines, implying ${ }^{15} \mathrm{~N}$-containing fragments would help verify the source and position of nitrogen atoms in N-DBPs (Figure S2D).

To ensure that the identified N-DBPs through screening workflow were produced from chloramination reactions, ratios of ${ }^{15} \mathrm{~N}-\mathrm{DBP} /{ }^{14} \mathrm{~N}-\mathrm{DBP}$ chromatographic peak areas in all samples were continuously monitored and shown in Figure S5. All N-DBPs in the phenazine group (Phes), 5,10-dihydro-phenazine group (DiH-Phes), and coupling product (DiDPAs) group had reasonable ratios of ${ }^{15} \mathrm{~N}-\mathrm{DBP} /{ }^{14} \mathrm{~N}-\mathrm{DBP}$ s between 0.7 and 1.3 , but the ratios of NDPhA were found less than 0.7 at initial reaction ( 0.5 and 1 hours). Similar phenomenon was observed for HO-NDPhA, which was considered to be caused by existence of $0.5 \%{ }^{14} \mathrm{~N}-\mathrm{NDPhA}$ impurity in the DPA commercial standard or other unknown reaction pathways. To avoid effect of the abnormal ${ }^{14} \mathrm{~N}-\mathrm{NDPhA}$ and ${ }^{14} \mathrm{~N}-\mathrm{HO}-$ $\mathrm{NDPhA}$, the corresponding ${ }^{15} \mathrm{~N}$-peaks were used to calculate chromatographic peak areas (equation 3).

$\mathrm{A}_{\mathrm{NDPhA}}=\left(\mathrm{A}^{{ }^{5} \mathrm{~N}-\mathrm{NDPhA}}-\mathrm{A}^{{ }^{4} \mathrm{~N}-\mathrm{NDPhA}} \times 0.74 \%\right) \times 2$

$\mathrm{A}^{1{ }^{5} \mathrm{~N}-\mathrm{NDPhA}}$ and $\mathrm{A}^{4}{ }^{4} \mathrm{~N}-\mathrm{NDPhA}$ refer to the peak areas of ${ }^{15} \mathrm{~N}-\mathrm{NDPhA}$ and ${ }^{14} \mathrm{~N}-\mathrm{NDPhA}$, and $0.74 \%$ is natural abundance of ${ }^{15} \mathrm{~N}-\mathrm{NDPhA}$ in NDPhA.

\section{Appendix S5. Selection of Quenching Agents for N-chlorinated Products}

The reactivity of five reported quenching agents with free chlorine were in the following order: trimethoxybenzene $<$ ammonia $<$ ascorbic acid < sulfite < thiosulfate. ${ }^{\mathrm{S} 1}$ According to the reactivity, trimethoxybenzene, ammonium chloride and ascorbic acid were selected for trials to quench the N-chlorinated products. Three parallel experiments were performed by adding $2 \mathrm{mM}$ of quenching agents (trimethoxybenzene, ammonium chloride and ascorbic acid) to reaction solutions with monochloramine /DPA molar ratio of $100 / 1$ at $\mathrm{pH}=7$ for 12 hours. The analytes after the quenching procedure were then extracted through HLB cartridges within 30 min. 
After quenching with trimethoxybenzene and ammonium chloride, all chlorinated $\mathrm{N}$ DBPs were still detected. For instance, compared with the instrumental response of 9.6 $\times 10^{7}$ in unquenched sample, the IRs of Cl-DiH-Phe were still as high as $9.4 \times 10^{7}$ and $8.1 \times 10^{7}$ after ammonium chloride and trimethoxybenzene quenching procedure, respectively. In previous study, ${ }^{\mathrm{S} 2}$ trimethoxybenzene was able to quench free chlorine, but was insufficiently nucleophilic react with monochloramine. Ammonium chloride $\left(\mathrm{NH}_{4} \mathrm{Cl}\right)$ was also considered as a mild quenching agent of free chlorine, and did not react with $\mathrm{N}$-chlorinated products of organic compounds such as sulfamethoxazole and tramadol. ${ }^{\mathrm{S} 3 \mathrm{~S} 4}$ Therefore, ammonium chloride and trimethoxybenzene were not suitable quenching agents for the $\mathrm{N}$-chlorinated products in this study.

The reductive nature of ascorbic acid was utilized to identify $\mathrm{N}-\mathrm{Cl}$ bonds in chlorinated products of tyrosyl dipeptides. ${ }^{\mathrm{S} 5}$ It could also affect the stability of $\mathrm{C}-\mathrm{Cl}$ bond, but a continuous reaction time of 3 days was needed for effective degradation of chloropicrin. ${ }^{\text {S2 }}$ In our study, the quenching procedure (including the SPE pretreatment) were within merely $30 \mathrm{~min}$, which showed negligible impact on behaviors of the investigated substances in qualitative analysis. For instance, after quenching with ascorbic acid, IRs of the Cl-DiH-Phe with $\mathrm{N}-\mathrm{Cl}$ bond decreased under the instrumental detection limit, but IRs of $\mathrm{Cl}$-Phe with $\mathrm{C}-\mathrm{Cl}$ bond remained consistent (i.e. $1.9 \times 10^{6}$ and $2.0 \times 10^{6}$ with and without quenching procedure, respectively). It implied ascorbic acid could be used to distinguish $\mathrm{N}-\mathrm{Cl}$ and $\mathrm{C}-\mathrm{Cl}$ bond products in current study.

\section{Appendix S6. The Chloramination Experiment of Phenazine}

As commercial standard of phenazine (i.e. Phe) is available, additional chloramination experiment of Phe was performed by reaction of Phe with the chloramines, to check whether Cl-DiH-Phe could be formed from the Phe. Reaction conditions remained almost the same that $5 \mu \mathrm{M}$ of Phe were treated with $0.5 \mathrm{mM}$ chloramines at $\mathrm{pH}=7$ for 12 hours. No Cl-DiH-Phe was detected, and proportion of the dosed Phe remained constant (i.e. $99.1 \pm 0.7 \%$ ). Trace amounts of Cl-Phe (proportion: $0.01 \%$ ) and HO-Phe 
(proportion: $0.08 \%$ ) were also detected. The results might suggest formation of $\mathrm{Cl}-$ DiH-Phe was not from Phe, but from precursors such as DPA and other transit intermediate as shown in Figure 2 and Figure S7.

\section{References}

S1. Schreiber, I. M.; Mitch, W. A., Influence of the order of reagent addition on NDMA formation during chloramination. Environ. Sci. Technol. 2005, 39, 3811-3818. DOI: $10.1021 / \mathrm{es} 0483286$

S2. Lau, S. S.; Dias, R. P.; Martin-Culet, K. R.; Race, N. A.; Schammel, M. H.; Reber, K. P.; Roberts, A. L.; Sivey, J. D. 1,3,5-Trimethoxybenzene (TMB) as a new quenching agent for preserving redox-labile disinfection byproducts and for quantifying free chlorine and free bromine. Environ. Sci.: Water Res. Technol. 2018, 4, 926-941. DOI: 10.1039/C8EW00062J

S3. Dodd, M. C. and Huang, C. H. Transformation of the antibacterial agent sulfamethoxazole in reactions with chlorine: Kinetics, mechanisms, and pathways, Environ. Sci. Technol. 2004, 38, 5607-5615. DOI: 10.1021/es035225z

S4. Cheng, H. Song, D. Chang, Y. Liu, H. and Qu, J. Chlorination of tramadol: reaction kinetics, mechanism and genotoxicity evaluation, Chemosphere 2015, 141, 282-289. DOI: 10.1016/j.chemosphere.2015.06.034

S5. Huang, G.; Jiang, P.; Blackstock, L. K. J.; Tian, D. Y.; Li, X. F. Formation and occurrence of iodinated tyrosyl dipeptides in disinfected drinking water. Environ. Sci. Technol. 2018, 52, 4218-4226. DOI: 10.1021/acs.est.7b06276 
Table S1. Nitrosamines in carcinogen lists by International Agency for Research on Cancer, and nitrosamine DBPs in EPA's Drinking Water Contaminant Candidate List 4 (CCL 4) and Unregulated Contaminant Monitoring Rule 2 (UCMR 2).

\begin{tabular}{|c|c|c|c|c|}
\hline Name & Year & Group & CCL 4 & UCMR 2 \\
\hline N'-Nitrosonornicotine (NNN) & 2012 & 1 & & \\
\hline $\begin{array}{l}\text { 4-(N-Nitrosomethylamino)-1-(3-pyridyl)-1- } \\
\text { butanone (NNK) }\end{array}$ & 2012 & 1 & & \\
\hline $\begin{array}{l}\text { Semustine [1-(2-Chloroethyl)-3-(4- } \\
\text { methylcyclohexyl)-1-nitrosourea, Methyl-CCNU] }\end{array}$ & 2012 & 1 & & \\
\hline Bischloroethyl nitrosourea (BCNU) & 1987 & $2 \mathrm{~A}$ & & \\
\hline $\begin{array}{l}\text { 1-(2-Chloroethyl)-3-cyclohexyl-1-nitrosourea } \\
\text { (CCNU) }\end{array}$ & 1987 & $2 \mathrm{~A}$ & & \\
\hline N-Ethyl-N-nitrosourea & 1987 & $2 \mathrm{~A}$ & & \\
\hline N-Methyl-N'-nitro-N-nitrosoguanidine (MNNG) & 1987 & $2 \mathrm{~A}$ & & \\
\hline N-Methyl-N-nitrosourea & 1987 & $2 \mathrm{~A}$ & & \\
\hline N-Nitrosodiethylamine & 1987 & $2 \mathrm{~A}$ & $\mathrm{Y}$ & $\mathrm{Y}$ \\
\hline N-Nitrosodimethylamine & 1987 & $2 \mathrm{~A}$ & $\mathrm{Y}$ & $\mathrm{Y}$ \\
\hline N-Methyl-N-nitrosourethane & 1987 & $2 \mathrm{~B}$ & & \\
\hline N-Nitrosodiethanolamine & 2000 & 2B & & \\
\hline N-Nitrosodi-n-butylamine & 1987 & 2B & & $\mathrm{Y}$ \\
\hline N-Nitrosodi-n-propylamine & 1987 & $2 \mathrm{~B}$ & $\mathrm{Y}$ & $\mathrm{Y}$ \\
\hline 3-(N-Nitrosomethylamino)propionitrile & 2004 & $2 \mathrm{~B}$ & & \\
\hline N-Nitrosomethylethylamine & 1987 & $2 \mathrm{~B}$ & & $\mathrm{Y}$ \\
\hline N-Nitrosomethylvinylamine & 1987 & $2 \mathrm{~B}$ & & \\
\hline N-Nitrosomorpholine & 1987 & $2 \mathrm{~B}$ & & \\
\hline N-Nitrosopiperidine & 1987 & $2 \mathrm{~B}$ & & \\
\hline N-Nitrosopyrrolidine & 1987 & $2 \mathrm{~B}$ & $\mathrm{Y}$ & $\mathrm{Y}$ \\
\hline N-Nitrososarcosine & 1987 & 2B & & \\
\hline 3-(N-Nitrosomethylamino)propionaldehyde & 2004 & 3 & & \\
\hline N-Methyl-N,4-dinitrosoaniline & 1987 & 3 & & \\
\hline N'-Nitrosoanabasine (NAB) & 2007 & 3 & & \\
\hline N'-Nitrosoanatabine (NAT) & 2007 & 3 & & \\
\hline N-Nitrosodiphenylamine & 1987 & 3 & $\mathrm{Y}$ & \\
\hline N-Nitrosofolic acid & 1987 & 3 & & \\
\hline N-Nitrosoguvacine & 2004 & 3 & & \\
\hline N-Nitrosoguvacoline & 2004 & 3 & & \\
\hline N-Nitrosohydroxyproline & 1987 & 3 & & \\
\hline N-Nitrosoproline & 1987 & 3 & & \\
\hline
\end{tabular}


Table S2. Detailed information on CAS number, chemical name, annual production, molecular structure and formula of registered diaromatic amines in USA and EU.

\begin{tabular}{|c|c|c|c|c|c|c|c|}
\hline \multirow[b]{2}{*}{ CAS No. } & \multirow{2}{*}{ Chemical name } & \multicolumn{4}{|c|}{ Production (tonnes/year) } & \multirow{2}{*}{ Chemical structure } & \multirow{2}{*}{ Formula } \\
\hline & & USA Min & USA Max & EU Min & EU Max & & \\
\hline $122-39-4$ & Benzenamine, N-phenyl- & 22680 & 45359 & 10 & 100 & & $\mathrm{C} 12 \mathrm{H} 11 \mathrm{~N}$ \\
\hline $\begin{array}{c}64653- \\
59-4\end{array}$ & $\begin{array}{l}\text { Benzenamine, 2-ethyl-N-(2- } \\
\text { ethylphenyl)- }\end{array}$ & withheld & withheld & NR & NR & & $\mathrm{C} 16 \mathrm{H} 19 \mathrm{~N}$ \\
\hline $\begin{array}{c}41317- \\
15-1\end{array}$ & $\begin{array}{l}\text { Benzenamine, 4-methoxy-2- } \\
\text { methyl-N-phenyl- }\end{array}$ & withheld & withheld & 1 & 10 & & $\mathrm{C} 14 \mathrm{H} 15 \mathrm{NO}$ \\
\hline $\begin{array}{c}41374- \\
20-3\end{array}$ & $\begin{array}{c}\text { Benzenamine, N-(2,4- } \\
\text { dimethylphenyl)-4-methoxy-2-m } \\
\text { ethyl- }\end{array}$ & withheld & withheld & NR & NR & & C16H19NO \\
\hline $101-67-7$ & $\begin{array}{l}\text { Benzenamine, 4-octyl-N-(4- } \\
\text { octylphenyl)- }\end{array}$ & 11 & 45 & NR & NR & & $\mathrm{C} 28 \mathrm{H} 43 \mathrm{~N}$ \\
\hline $\begin{array}{l}36878- \\
20-3\end{array}$ & $\begin{array}{l}\text { Benzenamine, ar-nonyl-N- } \\
\text { (nonylphenyl)- }\end{array}$ & 22680 & 45359 & 10000 & 100000 & & $\mathrm{C} 30 \mathrm{H} 47 \mathrm{~N}$ \\
\hline $\begin{array}{l}68608- \\
79-7\end{array}$ & $\begin{array}{l}\text { Benzenamine, N-phenyl-, } \\
\text { (tripropenyl) derivs. }\end{array}$ & withheld & withheld & NR & NR & Major components: mono/disubstituted & $\begin{array}{l}\mathrm{C} 21 \mathrm{H} 29 \mathrm{~N} \\
\mathrm{C} 30 \mathrm{H} 47 \mathrm{~N}\end{array}$ \\
\hline $\begin{array}{c}68411- \\
46-1\end{array}$ & $\begin{array}{l}\text { Benzenamine, } \mathrm{N} \text {-phenyl-, } \\
\text { reaction products with } 2,4,4- \\
\text { trimethylpentene }\end{array}$ & 4536 & 22680 & 10000 & 100000 & Major components: mono/disubstituted & $\begin{array}{l}\mathrm{C} 20 \mathrm{H} 27 \mathrm{~N} \\
\mathrm{C} 28 \mathrm{H} 43 \mathrm{~N}\end{array}$ \\
\hline
\end{tabular}




\begin{tabular}{|c|c|c|c|c|c|c|c|}
\hline $\begin{array}{l}15721- \\
78-5\end{array}$ & $\begin{array}{l}\text { Benzenamine, } 4-(1,1,3,3- \\
\text { tetramethylbutyl)-N-[4-( } 1,1,3,3- \\
\text { tetramethylbutyl)phenyl]- }\end{array}$ & 0 & 11 & 1000 & 10000 & & $\mathrm{C} 28 \mathrm{H} 43 \mathrm{~N}$ \\
\hline $\begin{array}{c}184378- \\
08-3\end{array}$ & $\begin{array}{l}\text { Benzenamine, } \mathrm{N} \text {-phenyl-, } \\
\text { reaction products with } \\
\text { isobutylene and } 2,4,4- \\
\text { trimethylpentene }\end{array}$ & withheld & withheld & NR & NR & Major components: mono/disubstituted & $\begin{array}{l}\text { C16H19N, } \\
\text { C20H27N, } \\
\text { C24H35N, } \\
\text { C28H43N }\end{array}$ \\
\hline $\begin{array}{c}68921- \\
45-9\end{array}$ & $\begin{array}{l}\text { Benzenamine, } \mathrm{N} \text {-phenyl-, } \\
\text { reaction products with styrene } \\
\text { and 2,4,4-trimethylpentene }\end{array}$ & 45 & 227 & NR & NR & Major components: mono/disubstituted & $\begin{array}{l}\mathrm{C} 20 \mathrm{H} 27 \mathrm{~N}, \\
\mathrm{C} 20 \mathrm{H} 19 \mathrm{~N}, \\
\mathrm{C} 28 \mathrm{H} 35 \mathrm{~N} \\
\mathrm{C} 28 \mathrm{H} 43 \mathrm{~N} \\
\mathrm{C} 28 \mathrm{H} 27 \mathrm{~N}\end{array}$ \\
\hline $\begin{array}{l}68442- \\
68-2\end{array}$ & $\begin{array}{l}\text { Benzenamine, N-phenyl-, } \\
\text { styrenated }\end{array}$ & 454 & 4536 & 100 & 1000 & Major components: mono/disubstituted & $\begin{array}{l}\mathrm{C} 20 \mathrm{H} 19 \mathrm{~N} \\
\mathrm{C} 28 \mathrm{H} 27 \mathrm{~N}\end{array}$ \\
\hline $\begin{array}{c}10081- \\
67-1\end{array}$ & $\begin{array}{l}\text { Benzenamine, 4-(1-methyl-1- } \\
\text { phenylethyl)-N-[4-(1-m ethyl-1- } \\
\text { phenylethyl)phenyl]- }\end{array}$ & 454 & 4536 & 100 & 1000 & & $\mathrm{C} 30 \mathrm{H} 31 \mathrm{~N}$ \\
\hline $\begin{array}{c}68608- \\
77-5\end{array}$ & $\begin{array}{c}\text { Benzenamine, 2-ethyl-N-(2- } \\
\text { ethylphenyl)-, (tripropenyl) } \\
\text { derivs. }\end{array}$ & withheld & withheld & NR & NR & Major components: mono/disubstituted & $\begin{array}{l}\mathrm{C} 25 \mathrm{H} 37 \mathrm{~N} \\
\mathrm{C} 34 \mathrm{H} 45 \mathrm{~N}\end{array}$ \\
\hline $90-30-2$ & 1-Naphthalenamine, N-phenyl- & 454 & 4536 & 100 & 1000 & & $\mathrm{C} 16 \mathrm{H} 13 \mathrm{~N}$ \\
\hline $\begin{array}{c}68259- \\
36-9\end{array}$ & $\begin{array}{l}\text { 1-Naphthalenamine, N-phenyl-ar- } \\
\text { (1,1,3,3-tetramethylbutyl)- }\end{array}$ & 227 & 454 & NR & NR & & $\mathrm{C} 24 \mathrm{H} 29 \mathrm{~N}$ \\
\hline
\end{tabular}




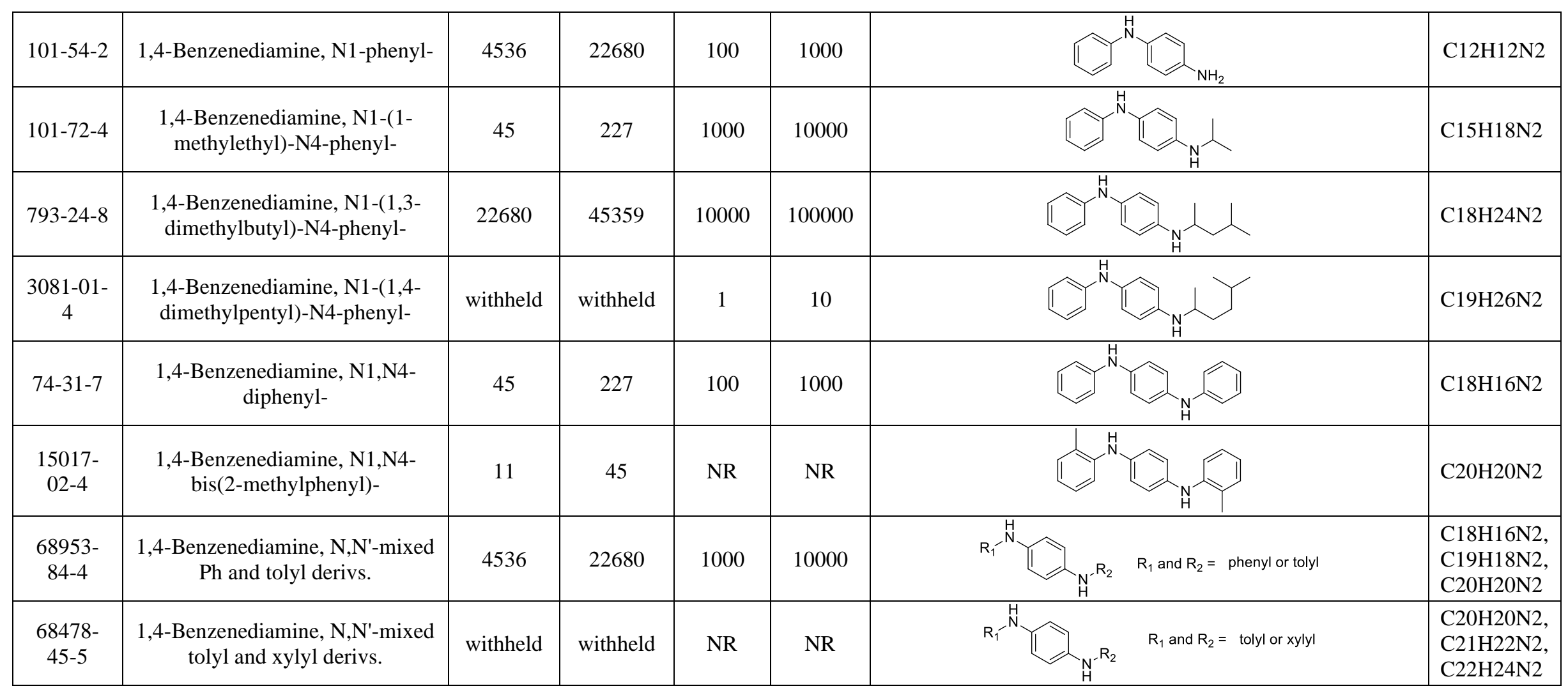


Table S3. Detailed information on CAS number, chemical name, annual production, molecular structure and formula of registered N-alkyl aromatic amines in USA and EU.

\begin{tabular}{|c|c|c|c|c|c|c|c|}
\hline \multirow[b]{2}{*}{ CAS No. } & \multirow[b]{2}{*}{ Chemical name } & \multicolumn{4}{|c|}{ Production (tonnes/year) } & \multirow[b]{2}{*}{ Chemical structure } & \multirow[b]{2}{*}{ Formula } \\
\hline & & USA Min & USA Max & $\begin{array}{l}\text { EU } \\
\text { Min }\end{array}$ & $\begin{array}{l}\text { EU } \\
\text { Max }\end{array}$ & & \\
\hline $100-61-8$ & Benzenamine, N-methyl- & withheld & withheld & NR & NR & & C7H9N \\
\hline $623-08-5$ & Benzenamine, N,4-dimethyl- & withheld & withheld & NR & NR & & $\mathrm{C} 8 \mathrm{H} 11 \mathrm{~N}$ \\
\hline $\begin{array}{c}56038-89- \\
2\end{array}$ & Benzenamine, N-(1-ethylpropyl)-3,4-dimethyl- & withheld & withheld & NR & NR & & $\mathrm{C} 13 \mathrm{H} 21 \mathrm{~N}$ \\
\hline $3068-76-6$ & Benzenamine, N-[3-(trimethoxysilyl)propyl]- & withheld & withheld & NR & NR & & $\begin{array}{c}\mathrm{C} 12 \mathrm{H} 19 \mathrm{SiO} 3 \\
\mathrm{~N}\end{array}$ \\
\hline $5285-60-9$ & Benzenamine, 4,4'-methylenebis[N-(1-methylpropyl)- & withheld & withheld & NR & NR & & $\mathrm{C} 21 \mathrm{H} 30 \mathrm{~N} 2$ \\
\hline $2051-79-8$ & $\begin{array}{l}\text { 1,4-Benzenediamine, N4,N4-diethyl-2-methyl-, } \\
\text { hydrochloride (1:1) }\end{array}$ & withheld & withheld & NR & NR & & $\mathrm{C} 11 \mathrm{H} 18 \mathrm{~N} 2$ \\
\hline $\begin{array}{l}63302-43- \\
2\end{array}$ & 1,4-Benzenediamine, N1-(1,4-dimethylpentyl)- & $\begin{array}{l}91(2011 \\
\text { year) }\end{array}$ & $\begin{array}{l}91(2011 \\
\text { year })\end{array}$ & NR & NR & & $\mathrm{C} 13 \mathrm{H} 22 \mathrm{~N} 2$ \\
\hline $101-96-2$ & 1,4-Benzenediamine, N1,N4-bis(1-methylpropyl)- & 454 & 4536 & 100 & 1000 & & $\mathrm{C} 14 \mathrm{H} 24 \mathrm{~N} 2$ \\
\hline $3081-14-9$ & 1,4-Benzenediamine, N1,N4-bis(1,4-dimethylpentyl)- & 454 & 4536 & 1000 & 10000 & & $\mathrm{C} 20 \mathrm{H} 36 \mathrm{~N} 2$ \\
\hline
\end{tabular}


Table S4. Detailed information on CAS number, chemical name, abbreviated name, formula, structure, and supplier of the target secondary amine precursors and nitrosamine analogues used in this study.

\begin{tabular}{|c|c|c|c|c|c|c|}
\hline $\begin{array}{l}\text { CAS } \\
\text { No. }\end{array}$ & Chemical name & $\begin{array}{l}\text { Abbreviated } \\
\text { name }\end{array}$ & Formula & Supplier & $\begin{array}{c}\mathrm{pK}_{\mathrm{b}} \\
\text { values }^{\mathrm{d}}\end{array}$ & Structure \\
\hline $\begin{array}{l}122-39- \\
4\end{array}$ & Diphenylamine & DPA & $\mathrm{C} 12 \mathrm{H} 11 \mathrm{~N}$ & $\mathrm{TCI}^{\mathrm{a}}$ & 13.2 & \\
\hline $\begin{array}{l}135-88- \\
6\end{array}$ & $\begin{array}{l}\text { N-Phenyl-2- } \\
\text { naphthylamine }\end{array}$ & NPNA & $\mathrm{C} 16 \mathrm{H} 13 \mathrm{~N}$ & TCI & $\begin{array}{c}\text { Not } \\
\text { searchable }\end{array}$ & \\
\hline $\begin{array}{l}10081- \\
67-1\end{array}$ & $\begin{array}{l}4,4 \text { '-Bis }(1,1- \\
\text { dimethylbenzyl)diphen } \\
\text { ylamine }\end{array}$ & diAMS & $\mathrm{C} 30 \mathrm{H} 31 \mathrm{~N}$ & TCI & $\begin{array}{c}\text { Not } \\
\text { searchable }\end{array}$ & \\
\hline $\begin{array}{l}100-61- \\
8\end{array}$ & N-Methylaniline & $\mathrm{NMeA}$ & $\mathrm{C} 7 \mathrm{H} 9 \mathrm{~N}$ & TCI & 9.2 & \\
\hline $\begin{array}{l}103-69- \\
5\end{array}$ & N-Ethylaniline & NEtA & C8H11N & TCI & 8.7 & \\
\hline $\begin{array}{l}622-80- \\
0\end{array}$ & N-Propylaniline & $\mathrm{NPrA}$ & C9H13N & TCI & 8.9 & \\
\hline $\begin{array}{l}1126- \\
78-9\end{array}$ & N-Butylaniline & $\mathrm{NBuA}$ & $\mathrm{C} 10 \mathrm{H} 15 \mathrm{~N}$ & TCI & 8.9 & \\
\hline $\begin{array}{l}1821- \\
36-9\end{array}$ & N-Cyclohexylaniline & $\mathrm{NChA}$ & $\mathrm{C} 12 \mathrm{H} 17 \mathrm{~N}$ & TCI & 8.4 & \\
\hline $\begin{array}{l}109-89- \\
7\end{array}$ & Diethylamine & DEtA & $\mathrm{C} 4 \mathrm{H} 11 \mathrm{~N}$ & Aladdin & 2.9 & \\
\hline $\begin{array}{l}142-84- \\
7\end{array}$ & Dipropylamine & $\mathrm{DPrA}$ & C6H15N & TCI & 3.0 & \\
\hline $\begin{array}{l}111-92- \\
2\end{array}$ & Dibutylamine & $\mathrm{DBuA}$ & C8H19N & TCI & 2.7 & \\
\hline $\begin{array}{l}123-75- \\
1\end{array}$ & Pyrrolidine & Pyr & $\mathrm{C} 4 \mathrm{H} 9 \mathrm{~N}$ & TCI & 2.7 & \\
\hline $\begin{array}{l}110-91- \\
8\end{array}$ & Morpholine & Mor & $\mathrm{C} 4 \mathrm{H} 9 \mathrm{NO}$ & TCI & 5.3 & \\
\hline $\begin{array}{l}101-83- \\
7\end{array}$ & Dicyclohexylamine & DChA & $\mathrm{C} 12 \mathrm{H} 23 \mathrm{~N}$ & TCI & $\begin{array}{c}\text { Not } \\
\text { searchable }\end{array}$ & \\
\hline $55-18-5$ & N-Nitrosodiethylamine & NDEA & $\mathrm{C} 4 \mathrm{H} 10 \mathrm{~N} 2 \mathrm{O}$ & $\begin{array}{l}\text { Sigma- } \\
\text { Aldrich }\end{array}$ & - & \\
\hline $\begin{array}{l}\text { 621-64- } \\
7\end{array}$ & $\begin{array}{l}\mathrm{N}- \\
\text { Nitrosodipropylamine }\end{array}$ & NDPA & $\mathrm{C} 6 \mathrm{H} 14 \mathrm{~N} 2 \mathrm{O}$ & $\begin{array}{l}\text { Sigma- } \\
\text { Aldrich }\end{array}$ & - & \\
\hline $\begin{array}{l}924-16- \\
3\end{array}$ & N-Nitrosodibutylamine & NDBA & $\mathrm{C} 8 \mathrm{H} 18 \mathrm{~N} 2 \mathrm{O}$ & $\begin{array}{l}\text { Sigma- } \\
\text { Aldrich }\end{array}$ & - & \\
\hline $\begin{array}{l}930-55- \\
2\end{array}$ & N-Nitrosopyrrolidine & NPyr & $\mathrm{C} 4 \mathrm{H} 8 \mathrm{~N} 2 \mathrm{O}$ & $\begin{array}{l}\text { Sigma- } \\
\text { Aldrich }\end{array}$ & - & \\
\hline $59-89-2$ & N-Nitrosomorpholine & NMor & $\mathrm{C} 4 \mathrm{H} 8 \mathrm{~N} 2 \mathrm{O} 2$ & $\begin{array}{l}\text { Sigma- } \\
\text { Aldrich }\end{array}$ & - & \\
\hline $\begin{array}{l}947-92- \\
2\end{array}$ & $\begin{array}{l}\mathrm{N}- \\
\text { Nitrosodicyclohexylam } \\
\text { ine }\end{array}$ & - & $\mathrm{C} 12 \mathrm{H} 22 \mathrm{~N} 2 \mathrm{O}$ & $\mathrm{TRC}^{\mathrm{c}}$ & - & \\
\hline $\begin{array}{l}614-00- \\
6\end{array}$ & $\begin{array}{l}\mathrm{N}-\mathrm{Nitroso}-\mathrm{N}- \\
\text { methylaniline }\end{array}$ & - & $\mathrm{C} 7 \mathrm{H} 8 \mathrm{~N} 2 \mathrm{O}$ & TCI & - & \\
\hline $\begin{array}{l}612-64- \\
6\end{array}$ & $\begin{array}{l}\text { N-Nitroso-N- } \\
\text { ethylaniline }\end{array}$ & - & $\mathrm{C} 8 \mathrm{H} 10 \mathrm{~N} 2 \mathrm{O}$ & $\mathrm{J} \& \mathrm{~K}$ & - & \\
\hline $86-30-6$ & $\begin{array}{l}\text { N- } \\
\text { Nitrosodiphenylamine }\end{array}$ & $\mathrm{NDPhA}$ & $\mathrm{C} 12 \mathrm{H} 22 \mathrm{~N} 2 \mathrm{O}$ & $\begin{array}{l}\text { Sigma- } \\
\text { Aldrich }\end{array}$ & - & \\
\hline $92-82-0$ & Phenazine & Phe & $\mathrm{C} 12 \mathrm{H} 8 \mathrm{~N} 2$ & TCI & - & \\
\hline
\end{tabular}

a Tokyo Chemical Industry Co., LTD. ${ }^{\mathrm{b}} \mathrm{J} \& \mathrm{~K}$ Scientific Ltd. ${ }^{\mathrm{c}}$ Toronto Research Chemicals Inc. ${ }^{\mathrm{d}}$ pK $\mathrm{b}$ values were from the CompTox Chemistry Dashboard, United State Environmental Protection Agency, https://comptox.epa.gov/dashboard (accessed November 21, 2019). 
Table S5. ${ }^{14} \mathrm{~N} /{ }^{15} \mathrm{~N}$-DBP pairs obtained from the N-DBPs screening workflow after reaction between DPA $(5 \mu \mathrm{M})$ and chloramines $(0.5 \mathrm{mM}$ with ${ }^{14} \mathrm{~N} /{ }^{15} \mathrm{~N}=1.0$ ) at $\mathrm{pH}=7$ for 12-hour treatment.

\begin{tabular}{|c|c|c|c|c|c|c|c|}
\hline \multirow{2}{*}{$\begin{array}{c}\mathrm{RT} \\
(\mathrm{min})\end{array}$} & \multicolumn{2}{|c|}{ Mass $[\mathrm{M}+\mathrm{H}]^{+}$} & \multirow{2}{*}{ Mass difference a $^{a}$} & \multirow{2}{*}{$\begin{array}{c}\text { Intensity } \\
\text { ratio }^{\mathrm{b}}\end{array}$} & \multirow{2}{*}{$\begin{array}{l}\text { Mass difference } \\
\text { Error (ppm) }\end{array}$} & \multirow{2}{*}{ Formula } & \multirow{2}{*}{ Abbreviated name } \\
\hline & ${ }^{14} \mathrm{~N}-\mathrm{DBPs}$ & ${ }^{15} \mathrm{~N}-\mathrm{DBPs}$ & & & & & \\
\hline 6.1 & 181.0759 & 182.0729 & 0.99701 & 0.98 & -0.18 & $\mathrm{C} 12 \mathrm{H} 8 \mathrm{~N} 2$ & Phe \\
\hline 6.7 & 183.0915 & 184.0886 & 0.99704 & 1.01 & 0.02 & $\mathrm{C} 12 \mathrm{H} 10 \mathrm{~N} 2$ & In-source fragment of Cl-DiH-Phe \\
\hline 7.9 & 183.0916 & 184.0886 & 0.99703 & 0.97 & -0.05 & $\mathrm{C} 12 \mathrm{H} 10 \mathrm{~N} 2$ & Quinonediimines (QDI) \\
\hline 5.5 & 185.1072 & 186.1043 & 0.99705 & 1.19 & 0.04 & $\mathrm{C} 12 \mathrm{H} 12 \mathrm{~N} 2$ & Aminodiphenylamine (NH2-DPA) \\
\hline 6.8 & 185.1073 & 186.1043 & 0.99701 & 0.93 & -0.15 & $\mathrm{C} 12 \mathrm{H} 12 \mathrm{~N} 2$ & NH2-DPA \\
\hline 5.7 & 197.0708 & 198.0678 & 0.99702 & 0.97 & -0.1 & $\mathrm{C} 12 \mathrm{H} 8 \mathrm{ON} 2$ & HO-Phe \\
\hline 6.3 & 199.0864 & 200.0835 & 0.99703 & 1.01 & -0.07 & $\mathrm{C} 12 \mathrm{H} 10 \mathrm{ON} 2$ & NDPhA \\
\hline 6.5 & 213.0657 & 214.0627 & 0.99706 & 1.01 & 0.07 & $\mathrm{C} 12 \mathrm{H} 8 \mathrm{O} 2 \mathrm{~N} 2$ & DiHO-Phe \\
\hline 7.1 & 215.0369 & 216.034 & 0.99703 & 0.96 & -0.06 & $\mathrm{C} 12 \mathrm{H} 7{ }^{35} \mathrm{ClN} 2$ & Cl-Phe \\
\hline 6.4 & 215.0813 & 216.0783 & 0.99702 & 0.99 & -0.07 & $\mathrm{C} 12 \mathrm{H} 10 \mathrm{O} 2 \mathrm{~N} 2$ & HO-NDPhA \\
\hline 6.7 & 217.0525 & 218.0495 & 0.99702 & 0.98 & -0.08 & $\mathrm{C} 12 \mathrm{H} 9^{35} \mathrm{ClN} 2$ & Cl-DiH-Phe \\
\hline 6.7 & 219.0495 & 220.0465 & 0.99699 & 1.02 & -0.23 & $\mathrm{C} 12 \mathrm{H} 9^{37} \mathrm{ClN} 2$ & Cl-isotope peak \\
\hline 7.3 & 231.0317 & 232.0288 & 0.99705 & 1.19 & 0.04 & $\mathrm{C} 12 \mathrm{H} 7{ }^{35} \mathrm{ClON} 2$ & Cl-HO-Phe \\
\hline 7.3 & 233.029 & 234.026 & 0.99704 & 0.82 & 0.01 & $\mathrm{C} 12 \mathrm{H} 7{ }^{37} \mathrm{ClON} 2$ & Cl-isotope peak \\
\hline 6 & 233.0473 & 234.0444 & 0.99703 & 0.98 & -0.06 & $\mathrm{C} 12 \mathrm{H} 9^{35} \mathrm{ClON} 2$ & Cl-HO-DiH-Phe \\
\hline 6 & 235.0444 & 236.0414 & 0.99704 & 1.05 & -0.01 & $\mathrm{C} 12 \mathrm{H} 9^{37} \mathrm{Cl} \mathrm{ON} 2$ & Cl-isotope peak \\
\hline 6.4 & 235.0444 & 236.0415 & 0.99705 & 0.94 & 0.04 & $\mathrm{C} 12 \mathrm{H} 9^{37} \mathrm{Cl} \mathrm{ON} 2$ & Cl-isotope peak of Cl-NDPhA \\
\hline 7.2 & 251.0135 & 252.0105 & 0.99705 & 0.98 & 0.02 & $\mathrm{C} 12 \mathrm{H} 8^{35} \mathrm{Cl} 2 \mathrm{~N} 2$ & DiCl-DiH-Phe \\
\hline 7.2 & 253.0105 & 254.0076 & 0.99703 & 0.93 & -0.04 & $\mathrm{C} 12 \mathrm{H} 8^{35} \mathrm{Cl}^{37} \mathrm{ClN} 2$ & Cl-isotope peak \\
\hline 5.4 & 283.0032 & 284.0003 & 0.99705 & 0.91 & 0.04 & $\mathrm{C} 12 \mathrm{H} 88^{35} \mathrm{Cl} 2 \mathrm{O} 2 \mathrm{~N} 2$ & DiCl-DiHO-DiH-Phe \\
\hline 5.9 & 348.1489 & 349.146 & 0.99705 & 0.93 & 0.04 & $\mathrm{C} 24 \mathrm{H} 17 \mathrm{~N} 3$ & Unknown product \\
\hline 8.5 & 348.149 & 349.1461 & 0.99708 & 1.06 & 0.12 & $\mathrm{C} 24 \mathrm{H} 17 \mathrm{~N} 3$ & Cyc-DiDPA \\
\hline 9.2 & 348.1491 & 349.1461 & 0.99705 & 0.93 & 0.03 & $\mathrm{C} 24 \mathrm{H} 17 \mathrm{~N} 3$ & In-source fragment of Cl-Cyc-DiDPA \\
\hline 8.3 & 350.1646 & 351.1618 & 0.99711 & 0.92 & 0.21 & $\mathrm{C} 24 \mathrm{H} 19 \mathrm{~N} 3$ & DiDPA \\
\hline 8.7 & 366.1595 & 367.1567 & 0.99711 & 0.94 & 0.2 & C24H19ON3 & HO-DiDPA \\
\hline 9.2 & 382.11 & 383.107 & 0.99706 & 0.99 & 0.06 & $\mathrm{C} 24 \mathrm{H} 16^{35} \mathrm{ClN} 3$ & Cl-Cyc-DiDPA \\
\hline 7.7 & 382.11 & 383.1071 & 0.99706 & 0.92 & 0.05 & $\mathrm{C} 24 \mathrm{H} 16^{35} \mathrm{ClN} 3$ & Cl-Cyc-DiDPA \\
\hline 9.7 & 384.1256 & 385.1226 & 0.99701 & 0.97 & -0.07 & $\mathrm{C} 24 \mathrm{H} 18^{35} \mathrm{ClN} 3$ & Cl-DiDPA \\
\hline 8.7 & 384.1257 & 385.1227 & 0.99704 & 0.91 & 0 & $\mathrm{C} 24 \mathrm{H} 18^{35} \mathrm{ClN} 3$ & Cl-DiDPA \\
\hline 8.1 & 416.071 & 417.0679 & 0.99686 & 0.91 & -0.43 & $\mathrm{C} 24 \mathrm{H} 15^{35} \mathrm{Cl} 2 \mathrm{~N} 3$ & DiCl-Cyc-DiDPA \\
\hline 8.1 & 418.0678 & 419.0649 & 0.99711 & 0.91 & 0.18 & $\mathrm{C} 24 \mathrm{H} 15 \mathrm{~N}^{35} \mathrm{Cl}^{37} \mathrm{Cl}$ & Cl-isotope peak \\
\hline
\end{tabular}

${ }^{a}$ Mass difference of ${ }^{14} \mathrm{~N}$-DBPs and ${ }^{15} \mathrm{~N}$-DBPs equals to mass of ${ }^{15} \mathrm{~N}$-DBPs minus mass of ${ }^{14} \mathrm{~N}$-DBPs

${ }^{\mathrm{b}}$ Intensity ratio of ${ }^{14} \mathrm{~N}$-DBPs and ${ }^{15} \mathrm{~N}$-DBPs equals to intensity of ${ }^{15} \mathrm{~N}$-DBPs divided by intensity of ${ }^{14} \mathrm{~N}-\mathrm{DBPs}$ 
Table S6. Detailed information on characteristic $\mathrm{MS}^{2}$ fragment ions, existence of $\mathrm{N}-\mathrm{Cl}$ bond, and structure elucidation confidence level of the identified N-DBPs in chloramination of DPA.

\begin{tabular}{|c|c|c|c|c|c|c|}
\hline Name & $\begin{array}{l}\text { Retention } \\
\text { time (min) }\end{array}$ & $\begin{array}{l}\text { Collision } \\
\text { energy }(\%)^{\mathrm{a}}\end{array}$ & $\begin{array}{c}\text { Formula of } \\
\text { precursor ions } \\
\left([\mathrm{M}+\mathrm{H}]^{+}\right) \\
\end{array}$ & $\begin{array}{l}\text { Mass of fragment ions } \\
\text { (their formula and deviation) }\end{array}$ & $\begin{array}{l}\text { Type of } \\
\text { chlorine } \\
\text { bond }^{\text {b }}\end{array}$ & $\begin{array}{l}\text { Confidence } \\
\text { levels }{ }^{c}\end{array}$ \\
\hline \multirow[b]{2}{*}{ Phe } & \multirow[b]{2}{*}{6.07} & \multirow[b]{2}{*}{70} & {$\left[\mathrm{C}_{12} \mathrm{H}_{9}{ }^{14} \mathrm{~N}_{2}\right]^{+}$} & $\begin{array}{l}154.0652\left(\left[\mathbf{C}_{11} \mathbf{H}_{8}{ }^{\mathbf{1 4}} \mathbf{N}\right]^{+}, \mathbf{0 . 2 9} \mathbf{~ p p m}\right) \\
77.0388\left(\left[\mathbf{C}_{6} \mathbf{H}_{5}\right]^{+}, \mathbf{2 . 3 4} \mathbf{~ p p m}\right) \\
53.0388\left(\left[\mathbf{C}_{4} \mathbf{H}_{5}\right]^{+}, \mathbf{3 . 5 5} \mathbf{~ p p m}\right)\end{array}$ & \multirow[b]{2}{*}{-} & \multirow[b]{2}{*}{1} \\
\hline & & & {$\left[\mathrm{C}_{12} \mathrm{H}_{9}{ }^{14} \mathrm{~N}^{15} \mathrm{~N}\right]^{+}$} & $\begin{array}{l}155.0614\left(\left[\mathbf{C}_{11} \mathbf{H}_{8}{ }^{15} \mathbf{N}\right]^{+},-\mathbf{- 4 . 9 0} \mathbf{~ p p m}\right) \\
154.0654\left(\left[\mathbf{C}_{\mathbf{1 1}} \mathbf{H}_{8} \mathbf{8}^{\mathbf{1 4}} \mathbf{N}\right]^{+}, \mathbf{1 . 4 8} \mathbf{~ p p m}\right) \\
77.0388\left(\left[\mathbf{C}_{6} \mathbf{H}_{5}\right]^{+}, \mathbf{2 . 7 4} \mathbf{~ p p m}\right) \\
53.0388\left(\left[\mathbf{C}_{4} \mathbf{H}_{5}\right]^{+}, \mathbf{3 . 9 8} \mathbf{~ p p m}\right)\end{array}$ & & \\
\hline \multirow{2}{*}{ QDI } & \multirow{2}{*}{7.90} & \multirow{2}{*}{30} & {$\left[\mathrm{C}_{12} \mathrm{H}_{11}{ }^{14} \mathrm{~N}_{2}\right]^{+}$} & $\begin{array}{l}167.0731\left(\left[\mathbf{C}_{\mathbf{1 2}} \mathbf{H}_{\mathbf{9}^{\mathbf{1 4}}} \mathbf{N}\right]^{+}, \mathbf{0 . 6 5} \mathbf{p p m}\right) \\
166.0652\left(\left[\mathbf{C}_{\mathbf{1 2}} \mathbf{H}_{\mathbf{8}}{ }^{\mathbf{1 4}} \mathbf{N}\right]^{+}, \mathbf{0 . 5 7} \mathbf{p p m}\right) \\
156.0809\left(\left[\mathbf{C}_{\mathbf{1 1}} \mathbf{H}_{10}{ }^{\mathbf{1 4}} \mathbf{N}\right]^{+}, \mathbf{0 . 9 9} \mathbf{p p m}\right) \\
77.0388\left(\left[\mathbf{C}_{\mathbf{6}} \mathbf{H}_{5}\right]^{+}, \mathbf{2 . 6 4} \mathbf{p p m}\right) \\
\end{array}$ & \multirow[b]{2}{*}{-} & \multirow{2}{*}{3} \\
\hline & & & {$\left[\mathrm{C}_{12} \mathrm{H}_{11}{ }^{14} \mathrm{~N}^{15} \mathrm{~N}\right]^{+}$} & $\begin{array}{l}167.0731\left(\left[\mathbf{C}_{\mathbf{1 2}} \mathbf{H}_{\mathbf{9}^{14}} \mathbf{N}\right]^{+}, \mathbf{6 . 8 8} \mathbf{~ p p m}\right) \\
166.0652\left(\left[\mathbf{C}_{\mathbf{1 2}} \mathbf{H}_{\mathbf{8}}{ }^{\mathbf{1 4}} \mathbf{N}\right]^{+}, \mathbf{0 . 5 7} \mathbf{p p m}\right) \\
156.0808\left(\left[\mathbf{C}_{\mathbf{1 1}} \mathbf{H}_{\mathbf{1 0}}{ }^{\mathbf{1 4}} \mathbf{N}\right]^{+}, \mathbf{0 . 4 1} \mathbf{p p m}\right) \\
77.0388\left(\left[\mathbf{C}_{\mathbf{6}} \mathbf{H}_{5}\right]^{+}, \mathbf{2 . 2 3} \mathbf{p p m}\right) \\
\end{array}$ & & \\
\hline \multirow[b]{2}{*}{ NH2-DPA } & \multirow[b]{2}{*}{5.50} & \multirow[b]{2}{*}{30} & {$\left[\mathrm{C}_{12} \mathrm{H}_{13}{ }^{14} \mathrm{~N}_{2}\right]^{+}$} & $\begin{array}{l}168.0808\left(\left[\mathbf{C}_{12} \mathbf{H}_{10}{ }^{14} \mathbf{N}\right]^{+}, \mathbf{0 . 3 8} \mathbf{~ p p m}\right) \\
108.0684\left(\left[\mathbf{C}_{6} \mathbf{H}_{\mathbf{8}^{14}} \mathbf{N}_{2}\right]^{+}, \mathbf{2 . 2 2} \mathbf{p p m}\right) \\
93.0576\left(\left[\mathbf{C}_{6} \mathbf{H}_{7}{ }^{14} \mathbf{N}\right]^{+}, \mathbf{2 . 7 9} \mathbf{p p m}\right)\end{array}$ & \multirow[b]{2}{*}{-} & \multirow[b]{2}{*}{1} \\
\hline & & & {$\left[\mathrm{C}_{12} \mathrm{H}_{13}{ }^{14} \mathrm{~N}^{15} \mathrm{~N}\right]^{+}$} & $\begin{array}{l}168.0809\left(\left[\mathbf{C}_{\mathbf{1 2}} \mathbf{H}_{10}{ }^{14} \mathbf{N}\right]^{+}, \mathbf{1 . 0 4} \mathbf{~ p p m}\right) \\
\left.109.0654\left(\mathbf{C}_{\mathbf{6}} \mathbf{H}_{8}{ }^{\mathbf{1 4}} \mathbf{N}^{15} \mathbf{N}\right]^{+}, \mathbf{1 . 8 8} \mathbf{~ p p m}\right) \\
\left.94.0546\left(\mathbf{C}_{\mathbf{6}} \mathbf{H}_{7}{ }^{15} \mathbf{N}\right]^{+}, \mathbf{2 . 4 9} \mathbf{~ p p m}\right) \\
93.0575\left(\left[\mathbf{C}_{6} \mathbf{H}_{7}{ }^{\mathbf{1 4}} \mathbf{N}\right]^{+}, \mathbf{2 . 5 7} \mathbf{~ p p m}\right)\end{array}$ & & \\
\hline \multirow[b]{2}{*}{ NH2-DPA } & \multirow[b]{2}{*}{7.06} & \multirow[b]{2}{*}{30} & {$\left[\mathrm{C}_{12} \mathrm{H}_{13}{ }^{14} \mathrm{~N}_{2}\right]^{+}$} & $\begin{array}{l}168.0818\left(\left[\mathbf{C}_{12} \mathbf{H}_{10}{ }^{14} \mathbf{N}\right]^{+}, \mathbf{6 . 0 9} \mathbf{~ p p m}\right) \\
108.0684\left(\left[\mathbf{C}_{6} \mathbf{H}_{8}{ }^{\mathbf{1 4}} \mathbf{N}_{2}\right]^{+}, \mathbf{2 . 0 4} \mathbf{~ p p m}\right) \\
93.0575\left(\left[\mathbf{C}_{6} \mathbf{H}_{7}{ }^{\mathbf{1 4}} \mathbf{N}\right]^{+}, \mathbf{2 . 4 6} \mathbf{~ p p m}\right)\end{array}$ & \multirow[b]{2}{*}{-} & \multirow[b]{2}{*}{3} \\
\hline & & & {$\left[\mathrm{C}_{12} \mathrm{H}_{13}{ }^{14} \mathrm{~N}^{15} \mathrm{~N}\right]^{+}$} & $\begin{array}{l}168.0811\left(\left[\mathbf{C}_{\mathbf{1 2}} \mathbf{H}_{\mathbf{1 0}}{ }^{\mathbf{1 4}} \mathbf{N}\right]^{+}, \mathbf{1 . 6 3} \mathbf{~ p p m}\right) \\
\left.109.0654\left(\mathbf{C}_{\mathbf{6}} \mathbf{H}_{\mathbf{8}}{ }^{\mathbf{1 4}} \mathbf{N}^{15} \mathbf{N}\right]^{+}, \mathbf{1 . 8 8} \mathbf{~ p p m}\right) \\
94.0546\left(\mathbf{C}_{6} \mathbf{H}_{7}{ }^{15} \mathbf{N}^{+}, \mathbf{2 . 2 8} \mathbf{~ p p m}\right) \\
93.0575\left(\left[\mathbf{C}_{\mathbf{6}} \mathbf{H}_{7}{ }^{\mathbf{1 4}} \mathbf{N}\right]^{+}, \mathbf{2 . 4 6} \mathbf{~ p p m}\right)\end{array}$ & & \\
\hline \multirow{2}{*}{ HO-Phe } & \multirow{2}{*}{5.71} & \multirow{2}{*}{50} & {$\left[\mathrm{C}_{12} \mathrm{H}_{9} \mathrm{O}^{14} \mathrm{~N}_{2}\right]^{+}$} & $\begin{array}{l}\left.180.0682\left(\mathbf{C}_{12} \mathbf{H}_{8}{ }^{14} \mathbf{N}_{2}\right]^{+}, \mathbf{0 . 1 0} \mathbf{~ p p m}\right) \\
\left.169.0761\left(\mathbf{C}_{11} \mathbf{H}_{9}{ }^{14} \mathbf{N}_{2}\right]^{+}, \mathbf{0 . 2 9} \mathbf{~ p p m}\right) \\
\left.168.0683\left(\mathbf{C}_{\mathbf{1 1}} \mathbf{H}_{8}{ }^{14} \mathbf{N}_{2}\right]^{+}, \mathbf{0 . 6 0} \mathbf{~ p p m}\right)\end{array}$ & \multirow{2}{*}{-} & \multirow{2}{*}{3} \\
\hline & & & {$\left[\mathrm{C}_{12} \mathrm{H}_{9} \mathrm{O}^{14} \mathrm{~N}^{15} \mathrm{~N}\right]^{+}$} & 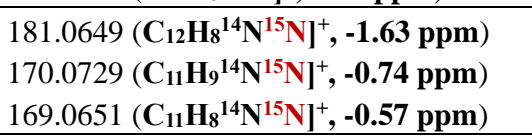 & & \\
\hline \multirow{2}{*}{ NDPhA } & \multirow{2}{*}{6.29} & \multirow{2}{*}{30} & {$\left[\mathrm{C}_{12} \mathrm{H}_{11} \mathrm{O}^{14} \mathrm{~N}_{2}\right]^{+}$} & $\begin{array}{l}169.0887\left(\left[\mathbf{C}_{\mathbf{1 2}} \mathbf{H}_{11}{ }^{14} \mathbf{N}\right]^{+}, \mathbf{0 . 7 6} \mathbf{~ p p m}\right) \\
\left.104.0497\left(\mathbf{C}_{7} \mathbf{H}_{6}{ }^{\mathbf{1 4}} \mathbf{N}\right]^{+}, \mathbf{2 . 3 5} \mathbf{~ p p m}\right) \\
66.0466\left(\left[\mathbf{C}_{5} \mathbf{H}_{6}\right]^{+}, \mathbf{3 . 5 8} \mathbf{~ p p m}\right)\end{array}$ & \multirow{2}{*}{-} & \multirow{2}{*}{1} \\
\hline & & & {$\left[\mathrm{C}_{12} \mathrm{H}_{11} \mathrm{O}^{14} \mathrm{~N}^{15} \mathrm{~N}\right]^{+}$} & $\begin{array}{l}169.0888\left(\left[\mathbf{C}_{\mathbf{1 2}} \mathbf{H}_{11}{ }^{14} \mathbf{N}\right]^{+}, \mathbf{1 . 2 1} \mathbf{~ p p m}\right) \\
104.0497\left(\left[\mathbf{C}_{7} \mathbf{H}_{6}{ }^{\mathbf{1 4}} \mathbf{N}\right]^{+}, \mathbf{2 . 2 8} \mathbf{~ p p m}\right) \\
66.0467\left(\left[\mathbf{C}_{5} \mathbf{H}_{6}\right]^{+}, \mathbf{3 . 5 8} \mathbf{~ p p m}\right) \\
\end{array}$ & & \\
\hline \multirow{2}{*}{ DiHO-Phe } & \multirow{2}{*}{6.46} & \multirow{2}{*}{30} & {$\left[\mathrm{C}_{12} \mathrm{H}_{9} \mathrm{O}_{2}{ }^{14} \mathrm{~N}_{2}\right]^{+}$} & $213.0659\left(\left[\mathbf{C}_{\mathbf{1 2}} \mathrm{H}_{\mathbf{8}} \mathbf{O}^{14} \mathbf{N}_{2}\right]^{+}, \mathbf{0 . 3 3} \mathbf{~ p p m}\right)$ & \multirow{2}{*}{-} & \multirow{2}{*}{3} \\
\hline & & & {$\left[\mathrm{C}_{12} \mathrm{H}_{9} \mathrm{O}_{2}{ }^{14} \mathrm{~N}^{15} \mathrm{~N}\right]^{+}$} & $214.0630\left(\left[\mathrm{C}_{12} \mathbf{H}_{8} \mathbf{O}^{14} \mathbf{N}^{15} \mathbf{N}\right]^{+},-\mathbf{1 . 4 7} \mathbf{~ p p m}\right)$ & & \\
\hline \multirow[b]{2}{*}{ Cl-Phe } & \multirow[b]{2}{*}{7.04} & & {$\left[\mathrm{C}_{12} \mathrm{H}_{8}{ }^{14} \mathrm{~N}_{2} \mathrm{Cl}\right]^{+}$} & $\begin{array}{l}180.0685\left(\left[\mathbf{C}_{\mathbf{1 2}} \mathbf{H}_{\mathbf{8}}{ }^{\mathbf{1 4}} \mathbf{N}_{\mathbf{2}}\right]^{+}, \mathbf{- 0 . 2 1} \mathbf{~ p p m}\right) \\
179.0604\left(\left[\mathbf{C}_{\mathbf{1 2}} \mathbf{H}_{7}{ }^{\mathbf{1 4}} \mathbf{N}_{\mathbf{2}}\right]^{+}, \mathbf{0 . 0 3} \mathbf{~ p p m}\right) \\
152.0495\left(\left[\mathbf{C}_{\mathbf{1 1}} \mathbf{H}_{6}{ }^{\mathbf{1 4}} \mathbf{N}\right]^{+}, \mathbf{0 . 2 6} \mathbf{~ p p m}\right) \\
75.0231\left(\left[\mathbf{C}_{\mathbf{6}} \mathbf{H}_{3}\right]^{+}, \mathbf{2 . 5 9} \mathbf{~ p p m}\right)\end{array}$ & & \\
\hline & & 70 & {$\left[\mathrm{C}_{12} \mathrm{H}_{8}{ }^{14} \mathrm{~N}^{15} \mathrm{NCl}\right]^{+}$} & $\begin{array}{l}181.0650\left(\left[\mathbf{C}_{12} \mathbf{H}_{8}{ }^{14} \mathbf{N}^{15} \mathbf{N}\right]^{+},-\mathbf{1 . 2 8} \mathbf{~ p p m}\right) \\
180.0572\left(\left[\mathbf{C}_{12} \mathbf{H}_{7}{ }^{\mathbf{1 4}} \mathbf{N}^{15} \mathbf{N}\right]^{+}, \mathbf{- 1 . 1 5} \mathbf{~ p p m}\right) \\
153.0467\left(\left[\mathbf{C}_{\mathbf{1 1}} \mathbf{H}_{6}{ }^{15} \mathbf{N}\right]^{+}, \mathbf{1 . 4 3} \mathbf{~ p p m}\right) \\
152.0496\left(\left[\mathbf{C}_{11} \mathbf{H}_{6}{ }^{\mathbf{1 4}} \mathbf{N}\right]^{+}, \mathbf{1 . 0 1} \mathbf{~ p p m}\right) \\
75.0231\left(\left[\mathbf{C}_{6} \mathbf{H}_{3}\right]^{+}, \mathbf{2 . 6 5} \mathbf{~ p p m}\right)\end{array}$ & $\mathrm{C}-\mathrm{Cl}$ bond & 3 \\
\hline $\begin{array}{c}\mathrm{HO}- \\
\text { NDPhA }\end{array}$ & 6.37 & 30 & {$\left[\mathrm{C}_{12} \mathrm{H}_{11} \mathrm{O}_{2}{ }^{14} \mathrm{~N}_{2}\right]^{+}$} & $\begin{array}{l}198.0789\left(\left[\mathbf{C}_{\mathbf{1 2}} \mathbf{H}_{\mathbf{1 0}} \mathbf{O}^{14} \mathbf{N}_{\mathbf{2}}\right]^{+}, \mathbf{0 . 6 2} \mathbf{~ p p m}\right) \\
168.0809\left(\left[\mathbf{C}_{\mathbf{1 2}} \mathbf{H}_{\mathbf{1 0}}{ }^{\mathbf{1 4}} \mathbf{N}\right]^{+}, \mathbf{0 . 8 4} \mathbf{~ p p m}\right)\end{array}$ & - & 3 \\
\hline
\end{tabular}




\begin{tabular}{|c|c|c|c|c|c|c|}
\hline & & & {$\left[\mathrm{C}_{12} \mathrm{H}_{11} \mathrm{O}_{2}{ }^{14} \mathrm{~N}^{15} \mathrm{~N}\right]^{+}$} & $\begin{array}{l}199.0755\left(\left[\mathbf{C}_{\mathbf{1 2}} \mathbf{H}_{10} \mathbf{O}^{\mathbf{1 4}} \mathbf{N}^{15} \mathbf{N}\right]^{+}, \mathbf{0 . 8 8} \mathbf{~ p p m}\right) \\
168.0810\left(\left[\mathbf{C}_{\mathbf{1 2}} \mathbf{H}_{\mathbf{1 0}}{ }^{\mathbf{1 4}} \mathbf{N}\right]^{+}, \mathbf{1 . 5 6} \mathbf{p p m}\right)\end{array}$ & & \\
\hline \multirow{2}{*}{$\begin{array}{l}\text { Cl-DiH- } \\
\text { Phe }\end{array}$} & \multirow{2}{*}{6.74} & \multirow{2}{*}{30} & {$\left[\mathrm{C}_{12} \mathrm{H}_{10}{ }^{14} \mathrm{~N}_{2} \mathrm{Cl}\right]^{+}$} & $\begin{array}{l}182.0839\left(\left[\mathbf{C}_{\mathbf{1 2}} \mathbf{H}_{\mathbf{1 0}}{ }^{\mathbf{1 4}} \mathbf{N}_{\mathbf{2}}\right]^{+}, \mathbf{0 . 3 3} \mathbf{~ p p m}\right) \\
181.0761\left(\left[\mathbf{C}_{\mathbf{1 2}} \mathbf{H}_{\mathbf{9}}{ }^{\mathbf{4}} \mathbf{N}_{\mathbf{2}}\right]^{+}, \mathbf{0 . 5 8} \mathbf{~ p p m}\right)\end{array}$ & \multirow{2}{*}{$\mathrm{N}-\mathrm{Cl}$ bond } & \multirow{2}{*}{$2 b$} \\
\hline & & & {$\left[\mathrm{C}_{12} \mathrm{H}_{10}{ }^{14} \mathrm{~N}^{15} \mathrm{NCl}\right]^{+}$} & $\begin{array}{l}183.0806\left(\left[\mathbf{C}_{12} \mathbf{H}_{10}{ }^{\mathbf{1 4}} \mathbf{N}^{15} \mathbf{N}\right]^{+}, \mathbf{- 1 . 3 4} \mathbf{~ p p m}\right) \\
182.0729\left(\left[\mathbf{C}_{\mathbf{1 2}} \mathbf{H}_{\mathbf{9}}{ }^{\mathbf{1 4}} \mathbf{N}^{15} \mathbf{N}\right]^{+}, \mathbf{- 0 . 9 9} \mathbf{~ p p m}\right) \\
\end{array}$ & & \\
\hline $\begin{array}{l}\text { Cl-HO- } \\
\text { Phe }\end{array}$ & 7.22 & - & - & - & C-Cl bond & 4 \\
\hline \multirow{2}{*}{$\begin{array}{l}\text { Cl- } \\
\text { NDPhA }\end{array}$} & \multirow[t]{2}{*}{6.33} & \multirow{2}{*}{30} & {$\left[\mathrm{C}_{12} \mathrm{H}_{10} \mathrm{O}^{14} \mathrm{~N}_{2} \mathrm{Cl}\right]^{+}$} & $\begin{array}{l}203.0497\left(\left[\mathbf{C}_{12} \mathbf{H}_{10}{ }^{14} \mathbf{N C l}\right]^{+}, \mathbf{0 . 5 7} \mathbf{p p m}\right) \\
168.0810\left(\left[\mathbf{C}_{12} \mathbf{H}_{10}{ }^{\mathbf{1 4}} \mathbf{N}\right]^{+}, \mathbf{1 . 1 1} \mathbf{~ p p m}\right) \\
66.0466\left(\left[\mathbf{C}_{5} \mathbf{H}_{6}\right]^{+}, \mathbf{3 . 4 7} \mathbf{~ p p m}\right)\end{array}$ & \multirow{2}{*}{ C-Cl bond } & \multirow{2}{*}{3} \\
\hline & & & {$\left[\begin{array}{c}{\left[\mathrm{C}_{12} \mathrm{H}_{10} \mathrm{O}^{14} \mathrm{~N}^{15} \mathrm{NCl}\right]} \\
+\end{array}\right.$} & $\begin{array}{l}203.0497\left(\left[\mathbf{C}_{12} \mathbf{H}_{10}{ }^{14} \mathbf{N C l}\right]^{+}, \mathbf{0 . 4 9} \mathbf{~ p p m}\right) \\
168.0809\left(\left[\mathbf{C}_{\mathbf{}_{2}} \mathbf{H}_{\mathbf{1 0}} \mathbf{1 0}^{\mathbf{1 4}} \mathbf{N}\right]^{+}, \mathbf{0 . 9 3} \mathbf{~ p p m}\right) \\
66.0466\left(\left[\mathbf{C}_{5} \mathbf{H}_{6}\right]^{+}, \mathbf{3 . 5 8} \mathbf{~ p p m}\right)\end{array}$ & & \\
\hline \multirow[b]{2}{*}{$\begin{array}{l}\text { Cl-OH- } \\
\text { DiH-Phe }\end{array}$} & \multirow[b]{2}{*}{6.00} & \multirow[b]{2}{*}{30} & {$\left[\mathrm{C}_{12} \mathrm{H}_{10} \mathrm{O}^{14} \mathrm{~N}_{2} \mathrm{Cl}\right]^{+}$} & $\begin{array}{l}197.0712\left(\left[\mathbf{C}_{12} \mathbf{H}_{9} \mathbf{O}^{14} \mathbf{N}_{2}\right]^{+}, \mathbf{1 . 5 3} \mathbf{~ p p m}\right) \\
170.0841\left(\left[\mathbf{C}_{11} \mathbf{H}_{\mathbf{1 0}} \mathbf{1}^{14} \mathbf{N}_{2}\right]^{+}, \mathbf{1 . 1 8} \mathbf{~ p p m}\right) \\
169.0762\left(\left[\mathbf{C}_{11} \mathbf{H}_{9^{14}} \mathbf{N}_{2}\right]^{+}, \mathbf{1 . 2 1} \mathbf{~ p p m}\right) \\
143.0732\left(\left[\mathbf{C}_{10} \mathbf{H}_{\mathbf{9}^{14}} \mathbf{N}\right]^{+}, \mathbf{1 . 6 0} \mathbf{~ p p m}\right) \\
\end{array}$ & \multirow[b]{2}{*}{$\mathrm{N}-\mathrm{Cl}$ bond } & \multirow[b]{2}{*}{3} \\
\hline & & & $\underset{+}{\left[\mathrm{C}_{12} \mathrm{H}_{10} \mathrm{O}^{14} \mathrm{~N}^{15} \mathrm{NCl}\right]}$ & 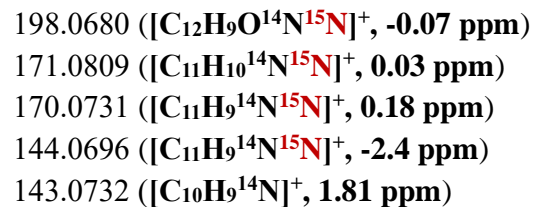 & & \\
\hline \multirow{2}{*}{$\begin{array}{l}\text { DiCl- } \\
\text { DiH-Phe }\end{array}$} & \multirow{2}{*}{7.13} & \multirow{2}{*}{30} & {$\left[\mathrm{C}_{12} \mathrm{H}_{9}{ }^{14} \mathrm{~N}_{2} \mathrm{Cl}_{2}\right]^{+}$} & $\begin{array}{l}216.0450\left(\left[\mathbf{C}_{12} \mathbf{H}_{9^{14}} \mathbf{N}_{2} \mathbf{C l}\right]^{+}, \mathbf{0 . 4 3} \mathbf{~ p p m}\right) \\
215.0372\left(\left[\mathbf{C}_{22} \mathbf{H}_{8}{ }^{14} \mathbf{N}_{2} \mathrm{Cl}\right]^{+}, \mathbf{0 . 6 4} \mathbf{~ p p m}\right) \\
181.0762\left(\left[\mathbf{C}_{\mathbf{1 2}} \mathbf{H}^{14}{ }^{14} \mathbf{N}_{2}\right]^{+}, \mathbf{0 . 9 1} \mathbf{~ p m m}\right)\end{array}$ & \multirow{2}{*}{$\begin{array}{l}\text { At least of } \\
\text { one } \mathrm{N}-\mathrm{Cl} \\
\text { bond }\end{array}$} & \multirow{2}{*}{3} \\
\hline & & & {$\left[\mathrm{C}_{12} \mathrm{H}_{9}{ }^{14} \mathrm{~N}^{15} \mathrm{NCl}_{2}\right]^{+}$} & 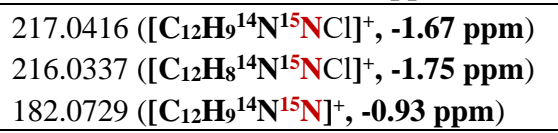 & & \\
\hline $\begin{array}{c}\text { DiCl- } \\
\text { DiHO- } \\
\text { DiH-Phe }\end{array}$ & 5.40 & - & - & - & $\begin{array}{l}\text { At least of } \\
\text { one } \mathrm{N}-\mathrm{Cl} \\
\text { bond }\end{array}$ & 4 \\
\hline \multirow{2}{*}{$\begin{array}{l}\text { Unkown } \\
\text { product }\end{array}$} & \multirow{2}{*}{5.98} & \multirow{2}{*}{70} & {$\left[\mathrm{C}_{24} \mathrm{H}_{18}{ }^{14} \mathrm{~N}_{3}\right]^{+}$} & 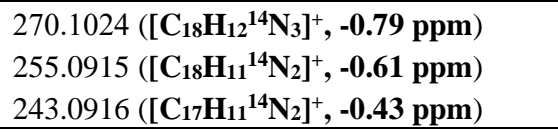 & \multirow[t]{2}{*}{ 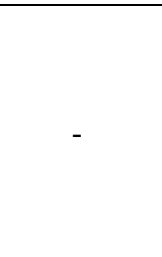 } & \multirow{2}{*}{4} \\
\hline & & & {$\left[\mathrm{C}_{24} \mathrm{H}_{18}{ }^{14} \mathrm{~N}_{2}{ }^{15} \mathrm{~N}\right]^{+}$} & 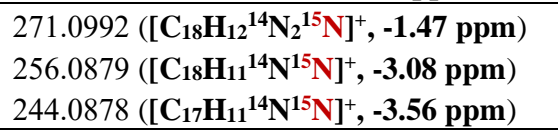 & & \\
\hline \multirow{2}{*}{$\begin{array}{l}\text { Cyc- } \\
\text { DiDPA }\end{array}$} & \multirow{2}{*}{8.34} & \multirow{2}{*}{70} & {$\left[\mathrm{C}_{24} \mathrm{H}_{18}{ }^{14} \mathrm{~N}_{3}\right]^{+}$} & 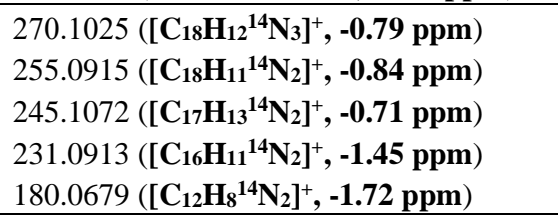 & \multirow[b]{2}{*}{ - } & \multirow{2}{*}{3} \\
\hline & & & {$\left[\mathrm{C}_{24} \mathrm{H}_{18}{ }^{14} \mathrm{~N}_{2}{ }^{15} \mathrm{~N}\right]^{+}$} & 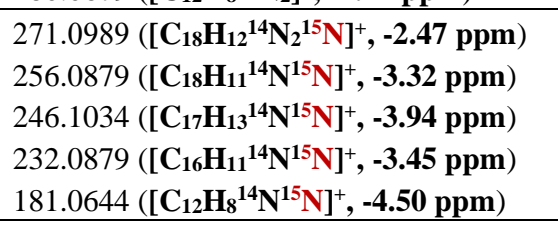 & & \\
\hline \multirow{2}{*}{ DiDPA } & \multirow{2}{*}{8.30} & \multirow{2}{*}{30} & {$\left[\mathrm{C}_{24} \mathrm{H}_{20}{ }^{14} \mathrm{~N}_{3}\right]^{+}$} & 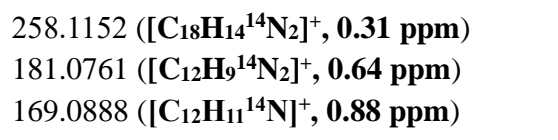 & \multirow{2}{*}{ - } & \multirow{2}{*}{$2 \mathrm{~b}$} \\
\hline & & & {$\left[\mathrm{C}_{24} \mathrm{H}_{20}{ }^{14} \mathrm{~N}_{2}{ }^{15} \mathrm{~N}\right]^{+}$} & 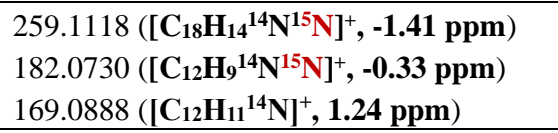 & & \\
\hline \multirow[t]{2}{*}{$\begin{array}{l}\text { HO- } \\
\text { DiDPA }\end{array}$} & 7.20 & 30 & {$\left[\mathrm{C}_{24} \mathrm{H}_{20} \mathrm{O}^{14} \mathrm{~N}_{3}\right]^{+}$} & 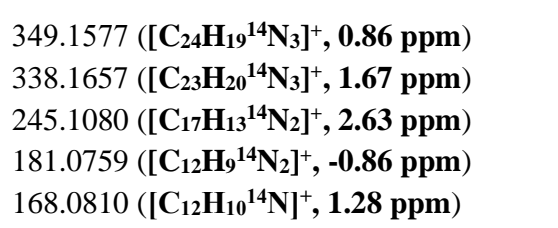 & - & 3 \\
\hline & & & {$\left[\mathrm{C}_{24} \mathrm{H}_{20} \mathrm{O}^{14} \mathrm{~N}_{2}{ }^{15} \mathrm{~N}\right]^{+}$} & $\begin{array}{l}350.1553\left(\left[\mathbf{C}_{\mathbf{2}} \mathbf{H} \mathbf{H}_{1}{ }^{\mathbf{1 4}} \mathbf{N}_{\mathbf{2}}{ }^{15} \mathbf{N}\right]^{+}, \mathbf{2 . 7 0} \mathbf{~ p p m}\right) \\
339.1629\left(\left[\mathbf{C}_{\mathbf{2} 3 \mathbf{H}} \mathbf{H}_{\mathbf{2 0}}{ }^{\mathbf{4}} \mathbf{N}_{\mathbf{2}}{ }^{15} \mathbf{N}\right]^{+}, \mathbf{2 . 1 3} \mathbf{~ p p m}\right)\end{array}$ & & \\
\hline
\end{tabular}




\begin{tabular}{|c|c|c|c|c|c|c|}
\hline & & & & $\begin{array}{l}246.1038\left(\left[\mathbf{C}_{17} \mathbf{H}_{13}{ }^{14} \mathbf{N}^{15} \mathbf{N}\right]^{+}, \mathbf{- 2 . 4 4} \mathbf{p p m}\right) \\
182.0731\left(\left[\mathbf{C}_{12} \mathbf{H}_{9}{ }^{14} \mathbf{N}^{15} \mathbf{N}\right]^{+}, \mathbf{0 . 0 6} \mathbf{~ p p m}\right) \\
168.0809\left(\left[\mathbf{C}_{12} \mathbf{H}_{10}{ }^{14} \mathbf{N}\right]^{+}, \mathbf{0 . 8 6} \mathbf{p p m}\right)\end{array}$ & & \\
\hline \multirow{2}{*}{$\begin{array}{l}\text { Cl-Cyc- } \\
\text { DiDPA }\end{array}$} & \multirow{2}{*}{9.18} & \multirow{2}{*}{70} & {$\left[\mathrm{C}_{24} \mathrm{H}_{17}{ }^{14} \mathrm{~N}_{3} \mathrm{Cl}\right]^{+}$} & 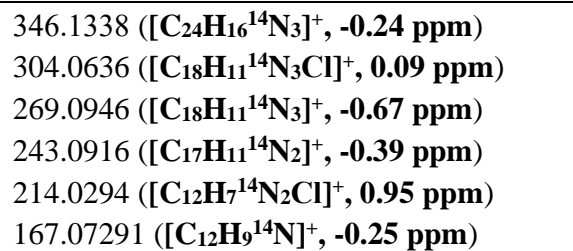 & \multirow{2}{*}{$\mathrm{C}-\mathrm{Cl}$ bond } & \multirow{2}{*}{3} \\
\hline & & & {$\left[\mathrm{C}_{24} \mathrm{H}_{17}{ }^{14} \mathrm{~N}_{2}{ }^{15} \mathrm{NCl}\right]^{+}$} & 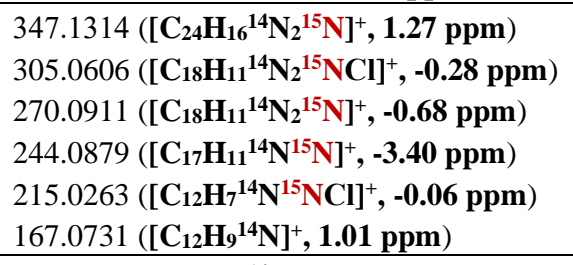 & & \\
\hline \multirow{2}{*}{$\begin{array}{l}\text { Cl-Cyc- } \\
\text { DiDPA }\end{array}$} & \multirow{2}{*}{7.52} & \multirow{2}{*}{70} & {$\left[\mathrm{C}_{24} \mathrm{H}_{17}{ }^{14} \mathrm{~N}_{3} \mathrm{Cl}\right]^{+}$} & 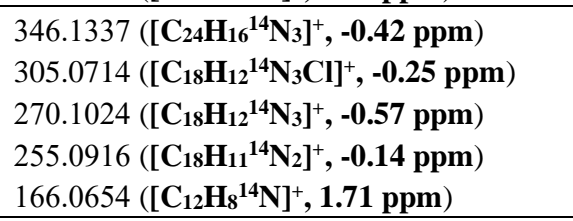 & \multirow{2}{*}{$\mathrm{N}-\mathrm{Cl}$ bond } & \multirow{2}{*}{3} \\
\hline & & & {$\left[\mathrm{C}_{24} \mathrm{H}_{17}{ }^{14} \mathrm{~N}_{2}{ }^{15} \mathrm{NCl}\right]^{+}$} & 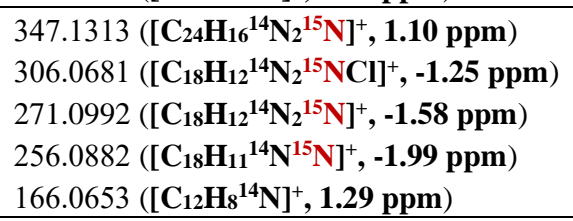 & & \\
\hline \multirow{2}{*}{ Cl-DiDPA } & \multirow[b]{2}{*}{9.70} & \multirow{2}{*}{30} & {$\left[\mathrm{C}_{24} \mathrm{H}_{19}{ }^{14} \mathrm{~N}_{3} \mathrm{Cl}\right]^{+}$} & $\begin{array}{l}348.1500\left(\left[\mathbf{C}_{24} \mathbf{H}_{18}{ }^{14} \mathbf{N}_{3}\right]^{+}, \mathbf{1 . 2 5} \mathbf{~ p p m}\right) \\
257.1074\left(\left[\mathbf{C}_{18} \mathbf{H}_{13}{ }^{1{ }^{14}} \mathbf{N}_{2}\right]^{+}, \mathbf{0 . 3 7} \mathbf{~ p p m}\right) \\
181.0767\left(\left[\mathbf{C}_{12} \mathbf{H}_{9}{ }^{14} \mathbf{N}_{\mathbf{2}}\right]^{+}, \mathbf{0 . 7 5} \mathbf{~ p p m}\right) \\
169.0888\left(\left[\mathbf{C}_{12} \mathbf{H}_{11}{ }^{14} \mathbf{N}^{+}, \mathbf{0 . 9 4} \mathbf{~ p p m}\right)\right.\end{array}$ & \multirow{2}{*}{$\mathrm{N}-\mathrm{Cl}$ bond } & \multirow{2}{*}{3} \\
\hline & & & {$\left[\mathrm{C}_{24} \mathrm{H}_{19}{ }^{14} \mathrm{~N}_{2}{ }^{15} \mathrm{NCl}\right]^{+}$} & $\begin{array}{l}349.1470\left(\left[\mathbf{C}_{24} \mathbf{H}_{18}{ }^{14} \mathbf{N}_{2}{ }^{15} \mathbf{N}\right]^{+}, \mathbf{1 . 1 8} \mathbf{~ p p m}\right) \\
258.1046\left(\left[\mathbf{C}_{18} \mathbf{H}_{13}{ }^{1{ }^{14}} \mathbf{N}^{15} \mathbf{N}\right]^{+}, \mathbf{0 . 8 5} \mathbf{~ p p m}\right) \\
182.0729\left(\left[\mathbf{C}_{12} \mathbf{H}_{9}{ }^{14} \mathbf{N}^{15} \mathbf{N}\right]^{+}, \mathbf{- 0 . 8 2} \mathbf{~ p p m}\right) \\
169.0889\left(\left[\mathbf{C}_{\mathbf{1 2}} \mathbf{H}_{11}{ }^{1{ }^{14}} \mathbf{N}\right]^{+}, \mathbf{1 . 9 5} \mathbf{~ p p m}\right)\end{array}$ & & \\
\hline \multirow{2}{*}{ Cl-DiDPA } & \multirow{2}{*}{8.59} & \multirow{2}{*}{30} & {$\left[\mathrm{C}_{24} \mathrm{H}_{19}{ }^{14} \mathrm{~N}_{3} \mathrm{Cl}\right]^{+}$} & $\begin{array}{l}348.1497\left(\left[\mathbf{C}_{\mathbf{2 4}} \mathbf{H}_{\mathbf{1 8}}{ }^{\mathbf{1 4}} \mathbf{N}_{\mathbf{3}}\right]^{+}, \mathbf{0 . 4 7} \mathbf{p p m}\right) \\
292.0763\left(\left[\mathbf{C}_{\mathbf{1 8}} \mathbf{H}_{\mathbf{1 3}} \mathbf{1}^{\mathbf{1 4}} \mathbf{N}_{\mathbf{2}} \mathbf{C l}\right]^{+}, \mathbf{0 . 3 9} \mathbf{~ p p m}\right) \\
257.1074\left(\left[\mathbf{C}_{\mathbf{1 8}} \mathbf{H}_{\mathbf{1 3}}{ }^{\mathbf{1 4}} \mathbf{N}_{\mathbf{2}}\right]^{+}, \mathbf{0 . 2 5} \mathbf{~ p p m}\right) \\
215.0372\left(\left[\mathbf{C}_{\mathbf{1 2}} \mathbf{H}_{\mathbf{8}}{ }^{\mathbf{1 4}} \mathbf{N}_{\mathbf{2}} \mathbf{C}\right]^{+}, \mathbf{0 . 8 3} \mathbf{~ p p m}\right) \\
181.0762\left(\left[\mathbf{C}_{\mathbf{1 2}} \mathbf{H}_{\mathbf{9}}{ }^{\mathbf{1 4}} \mathbf{N}_{\mathbf{2}}\right]^{+}, \mathbf{0 . 9 1} \mathbf{p p m}\right) \\
169.0889\left(\left[\mathbf{C}_{\mathbf{1 2}} \mathbf{H}_{\mathbf{1 1}}{ }^{\mathbf{1 4}} \mathbf{N}^{+}, \mathbf{1 . 4 7} \mathbf{p p m}\right)\right.\end{array}$ & \multirow{2}{*}{ C-Cl bond } & \multirow{2}{*}{3} \\
\hline & & & {$\left[\mathrm{C}_{24} \mathrm{H}_{19}{ }^{14} \mathrm{~N}_{2}{ }^{15} \mathrm{NCl}\right]^{+}$} & 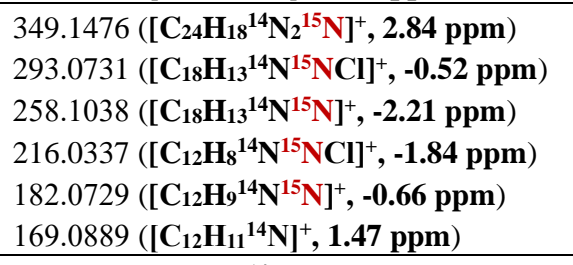 & & \\
\hline \multirow{2}{*}{$\begin{array}{l}\text { DiCl-Cyc- } \\
\text { DiDPA }\end{array}$} & \multirow{2}{*}{8.02} & \multirow{2}{*}{70} & {$\left[\mathrm{C}_{24} \mathrm{H}_{16}{ }^{14} \mathrm{~N}_{3} \mathrm{Cl}_{2}\right]^{+}$} & 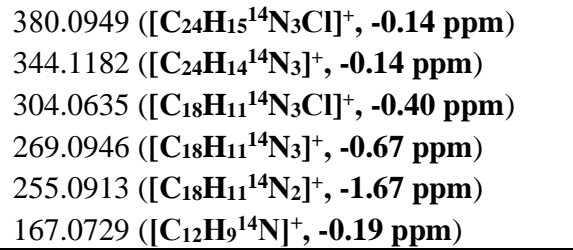 & \multirow{2}{*}{$\begin{array}{l}\text { At least of } \\
\text { one } \mathrm{N}-\mathrm{Cl} \\
\text { bond }\end{array}$} & \multirow{2}{*}{3} \\
\hline & & & $\underset{+}{\left[\mathrm{C}_{24} \mathrm{H}_{16}{ }^{14} \mathrm{~N}_{2}{ }^{15} \mathrm{NCl}_{2}\right]}$ & 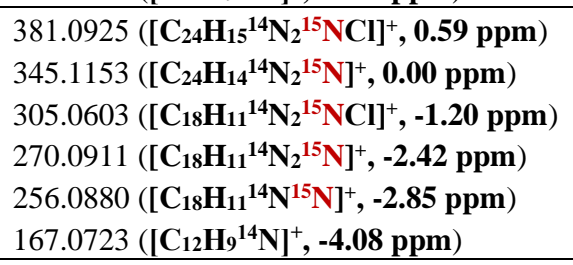 & & \\
\hline
\end{tabular}

${ }^{a}$ Collision energy is the energy of higher-energy collisional dissociation (HCD) mode.

${ }^{\mathrm{b}}$ Types of chlorine bond that include $\mathrm{N}-\mathrm{Cl}$ bond and $\mathrm{C}-\mathrm{Cl}$ bond, which were distinguished by ascorbic acid quenching experiment.

${ }^{c}$ Confidence level $=1,2,3$ and 4 represent confirmed structure by standards, probable structure by diagnostic evidence or library spectra, possible structures with positional isomers, and unknown structure with unequivocal molecular formula, respectively. The confirmed and proposed structures of N-DBPs were shown in Figure 1. 
Table S7. Effect of DOM (3.0 mg/L as C) and precursor concentrations on formation of Group I-IV N-DBPs after $0.5 \mathrm{mM}$ chloramine disinfection at $\mathrm{pH} 7$ for 12 hours.

\begin{tabular}{rccccc}
\hline & DPA & \multicolumn{4}{c}{ Molar yields (\%) $^{\mathrm{b}}$} \\
\cline { 3 - 6 } Samples & residual & Group I: & Group II: & Group III: & Group IV: \\
& $(\%)$ & NDPhAs & Phes & DiH-Phes & DiDPAs \\
\hline $5 \mu \mathrm{M}$ DPA & $3.0 \pm 0.2$ & $7.1 \pm 0.1$ & $13.9 \pm 0.5$ & $39.3 \pm 1.0$ & $5.1 \pm 0.1$ \\
$5 \mu \mathrm{M}$ DPA + DOM & $1.8 \pm 0.2$ & $17.2 \pm 0.7$ & $9.8 \pm 0.2$ & $19.1 \pm 0.2$ & $0.2 \pm 0.01$ \\
$0.5 \mu \mathrm{M}$ DPA + DOM & $2.3 \pm 0.1$ & $17.7 \pm 0.5$ & $9.9 \pm 0.6$ & $23.7 \pm 1.2$ & - \\
$0.05 \mu \mathrm{M}$ DPA + DOM & $3.3 \pm 0.4$ & $17.8 \pm 1.5$ & $7.9 \pm 0.5$ & $15.3 \pm 0.1$ & - \\
$0.005 \mu \mathrm{M}$ DPA + DOM & $-\mathrm{a}$ & $18.4 \pm 0.9$ & $6.7 \pm 0.5$ & $8.1 \pm 1.0$ & - \\
$0.0005 \mu \mathrm{M}$ DPA + DOM & - & - & $6.4 \pm 1.4$ & - & - \\
\hline
\end{tabular}

${ }^{a}$ No detected. ${ }^{b}$ Molar yields was obtained by semiquantitative analysis 
A

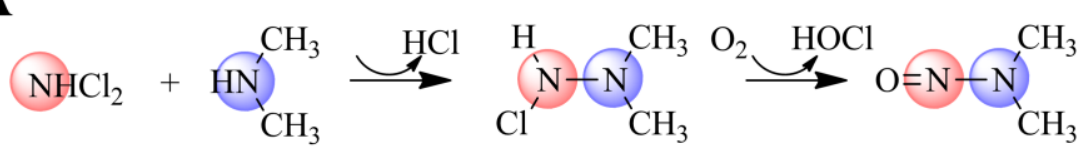

\section{B}
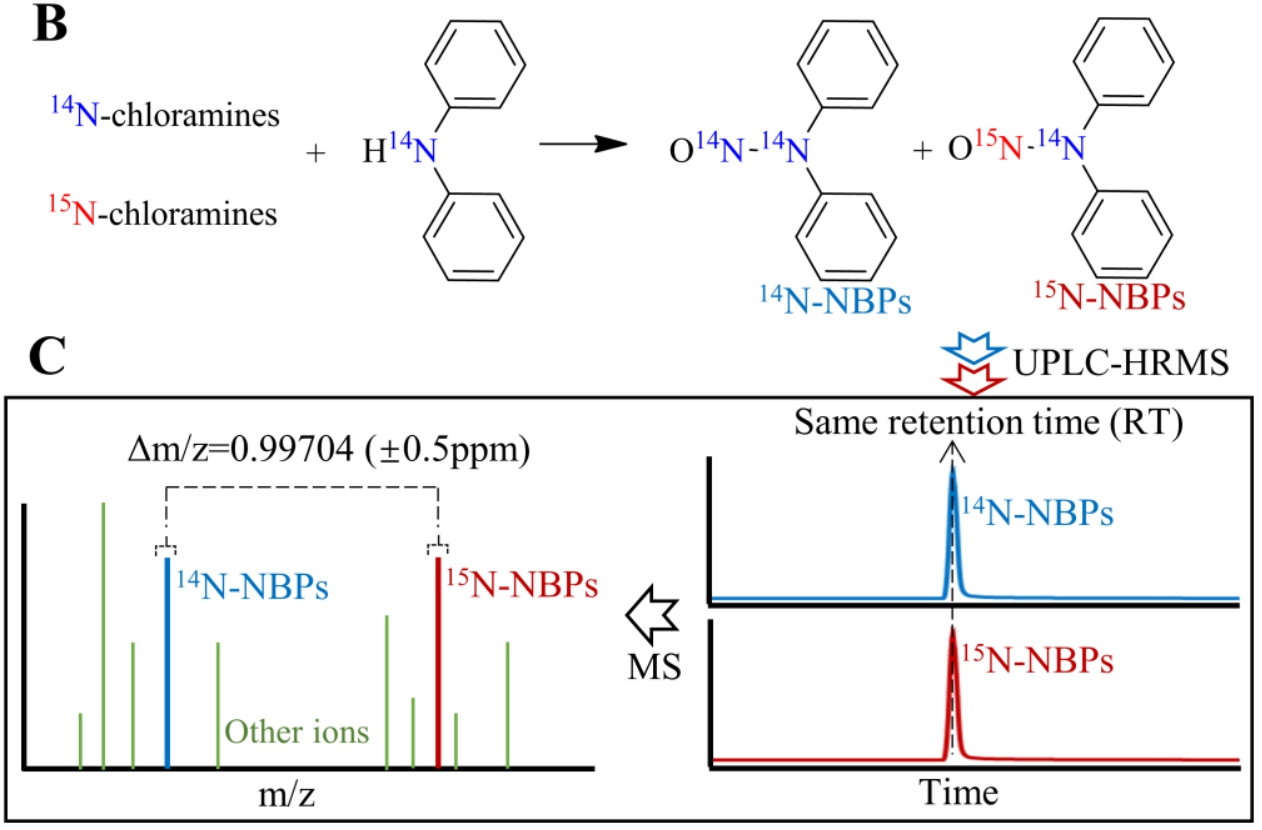

Figure S1. Scheme of N-DBP screening workflow. (A) Formation of NDMA through chlorinated unsymmetrical dimethylhydrazine intermediate in chloramination of DMA. (B) Theoretical formation process of ${ }^{14} \mathrm{~N}-\mathrm{NDPhA}$ and ${ }^{15} \mathrm{~N}-\mathrm{NDPhA}$ in chloramination of DPA with the equivalent molar amounts of ${ }^{14} \mathrm{~N}$-chloramines and ${ }^{15} \mathrm{~N}$-chloramines. (C) Theoretical chromatographic retention behaviors and ions signals of ${ }^{14} \mathrm{~N}$-DBPs and ${ }^{15} \mathrm{~N}$-DBPs. That is, ${ }^{14} \mathrm{~N}$-DBPs and ${ }^{15} \mathrm{~N}$-DBPs have the same retention time, and the corresponding ions have the same intensity as well as a mass difference of $0.99704( \pm$ 0.5 ppm Error Threshold). 


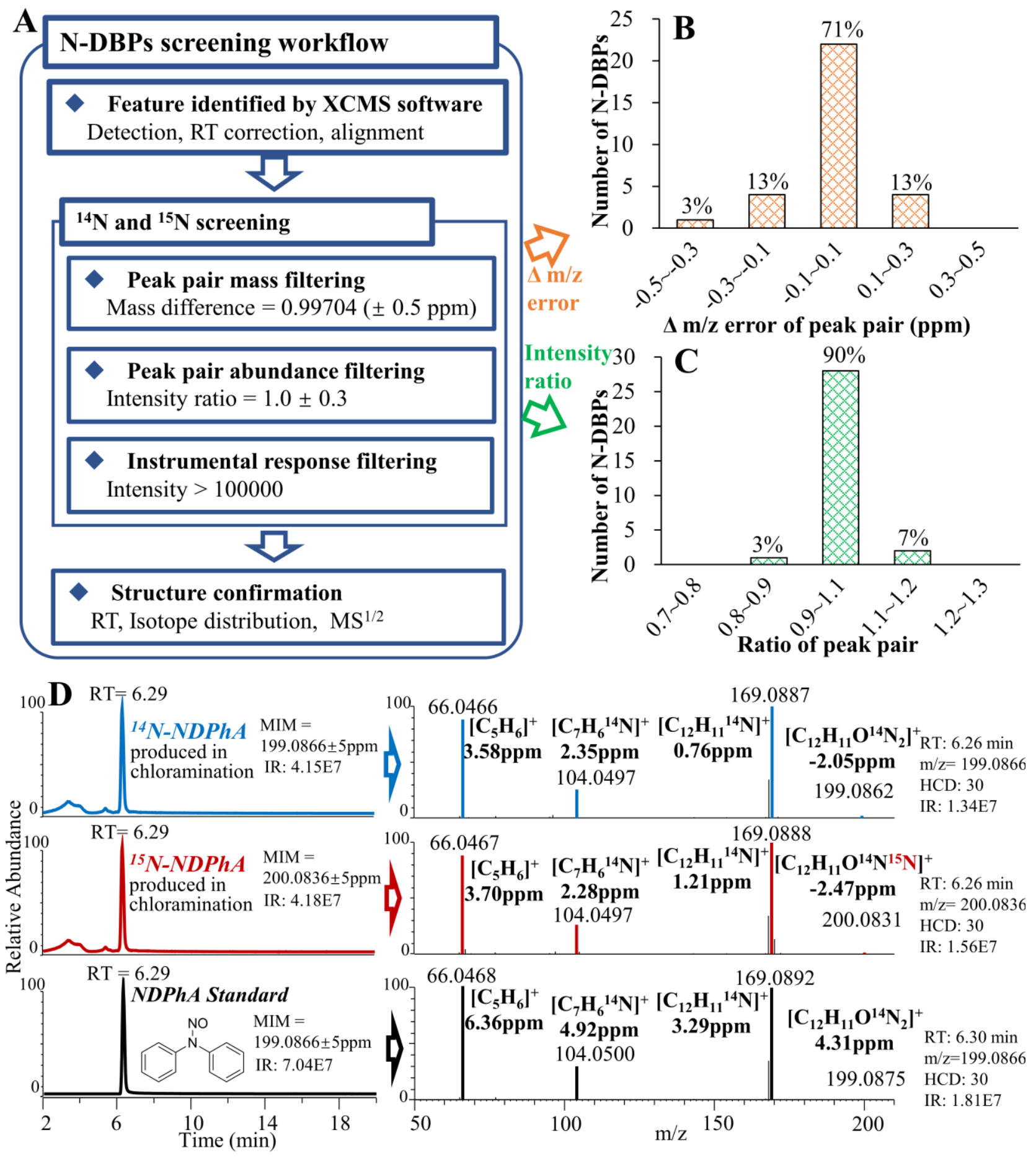

Figure S2. N-DBP screening workflow, and the method performance for identification of potential N-DBPs in chloramination of DPA. (A) Screening workflow for feature detection, peak pair filtering, and structure confirmation of N-DBPs. $(B)$ Distribution of the number of N-DBPs in which the mass differences of ${ }^{14} \mathrm{~N} /{ }^{15} \mathrm{~N}$-DBPs were within the range of $-0.5-0.5 \mathrm{ppm}$ (Error Threshold) compared with the theoretical value of 0.99704. (C) Distribution of the number of N-DBPs with intensity ratios of ${ }^{14} \mathrm{~N} /{ }^{15} \mathrm{~N}-\mathrm{DBPs}$ in the range of $1.0 \pm 0.3$. (D) The same retention behaviors and fragmentation patterns of ${ }^{14} \mathrm{~N} /{ }^{15} \mathrm{~N}-\mathrm{NDPhA}$ (blue and red) produced in chloramination and NDPhA standard (black), respectively. Data was from the reaction between DPA and chloramines with molar ratios of chloramines/precursor (100) at $\mathrm{pH} 7$ after $12 \mathrm{~h}$. 


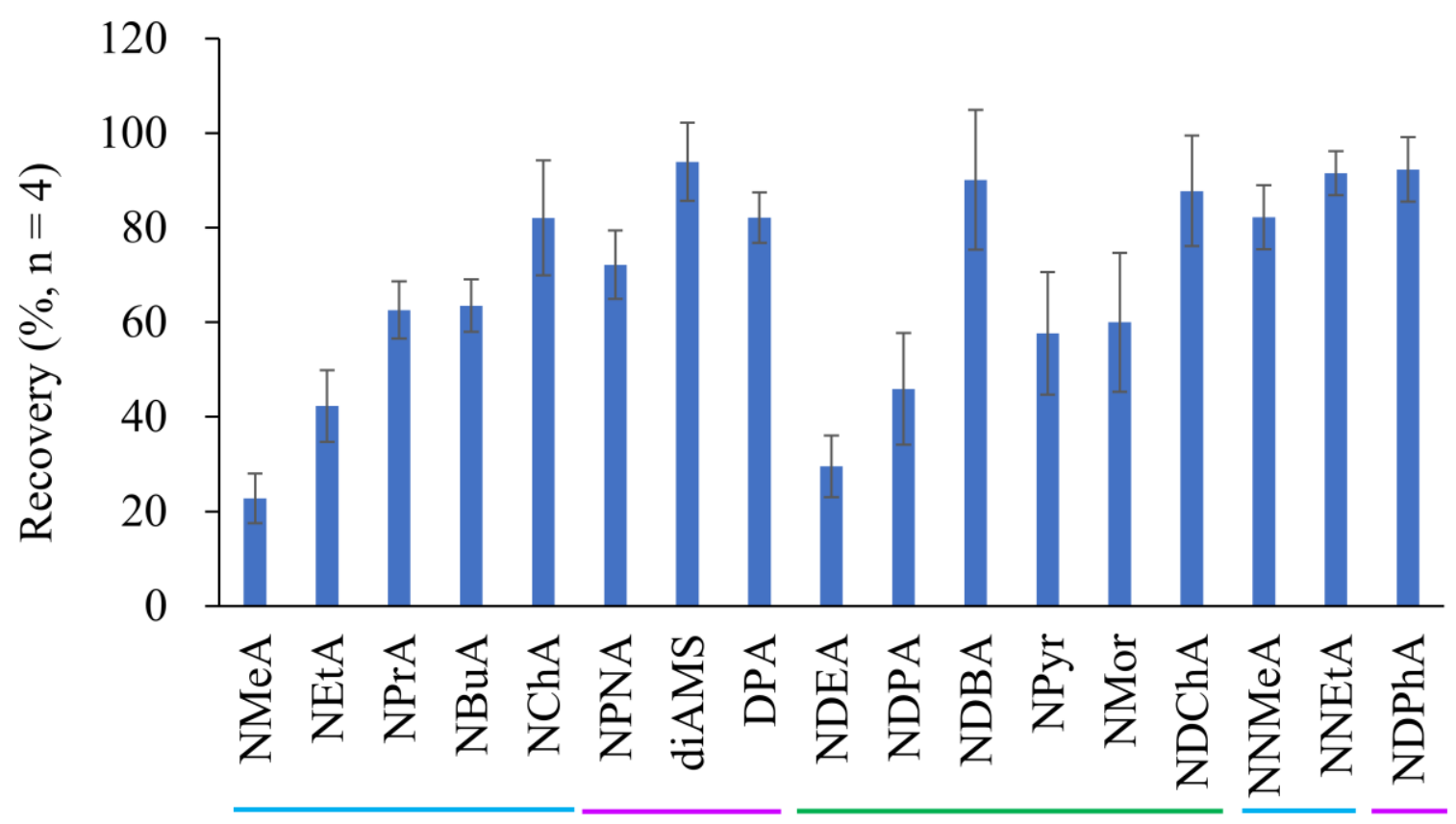

Figure S3. Recoveries of the targeted standards of diaromatic and N-alkyl aromatic secondary amine precursors and $\mathrm{N}$-nitrosamines $(10 \mathrm{nM}$ of spiked concentration for each analyte in $10 \mathrm{mM}$ phosphate buffer solution) by using the solid phase extraction method. 


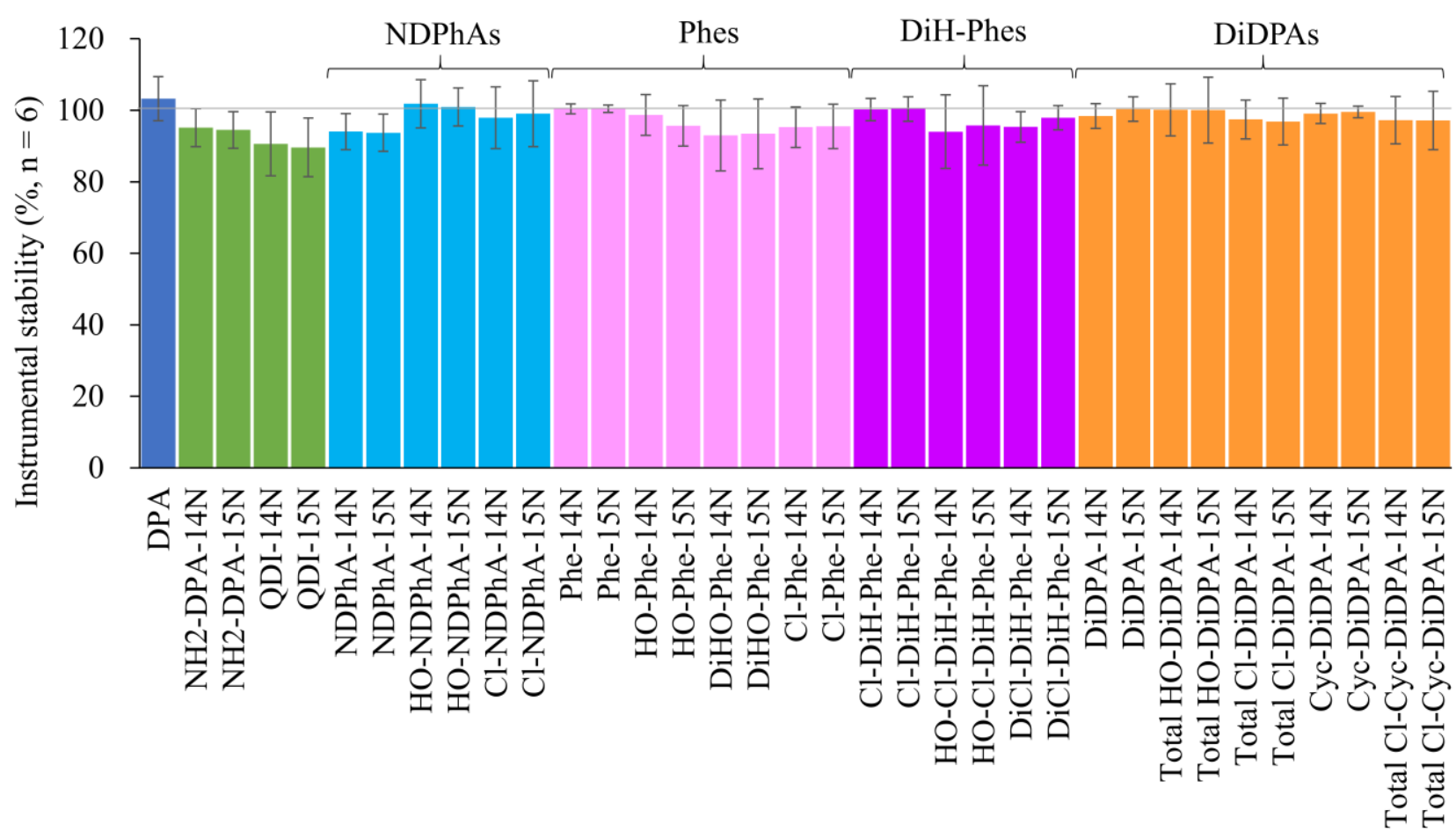

Figure S4. Stability of instrumental responses of the N-DBP analogues formed in the chloramination reactions by measuring quality control (QC) sample throughout batches of sample. The QC was prepared by mixing $10 \mu \mathrm{L}$ each sample in the experiments on chloramination of DPA at distinct disinfectant doses, $\mathrm{pH}$ values and reaction time. 

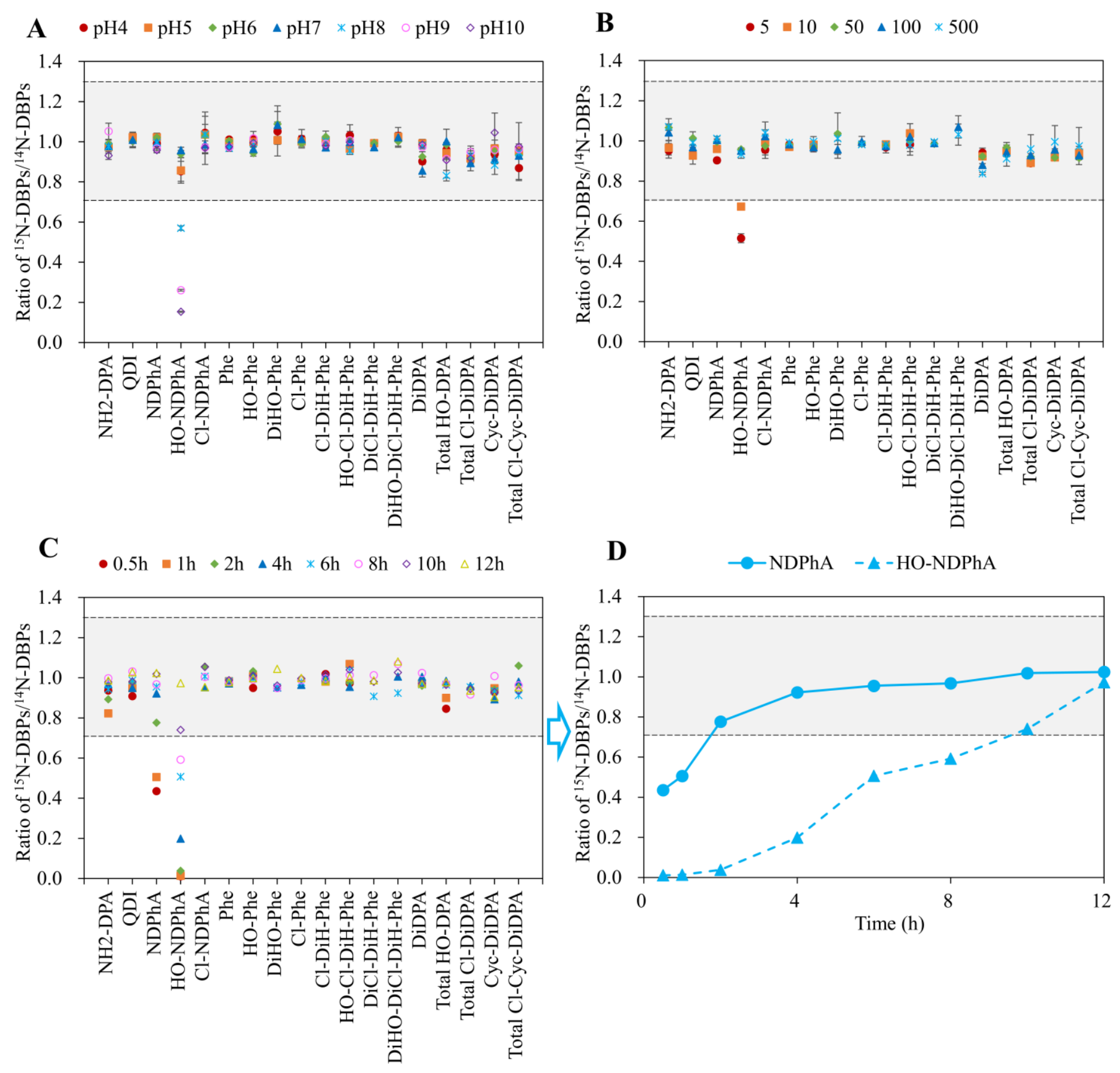

Figure S5. Ratios of ${ }^{15} \mathrm{~N}$-DBPs $/{ }^{14} \mathrm{~N}$-DBPs peak areas produced in chloramination of DPA with different reaction conditions, including $\mathrm{pH}$ values $(A)$, chloramines doses $(B)$ and reaction time $(C)$. Ratios of all $\mathrm{N}$ DBPs were within a reasonable range of 0.7 to 1.3, except for those of NDPhA and HO-NDPhA less than 0.7 under a few conditions. The ${ }^{15} \mathrm{~N}-\mathrm{DBP} /{ }^{14} \mathrm{~N}-\mathrm{DBP}$ peak area ratios of NDPhA and HO-NDPhA gradually approached 1.0 with the increase of reaction time shown in the dynamics diagram $(D)$. 

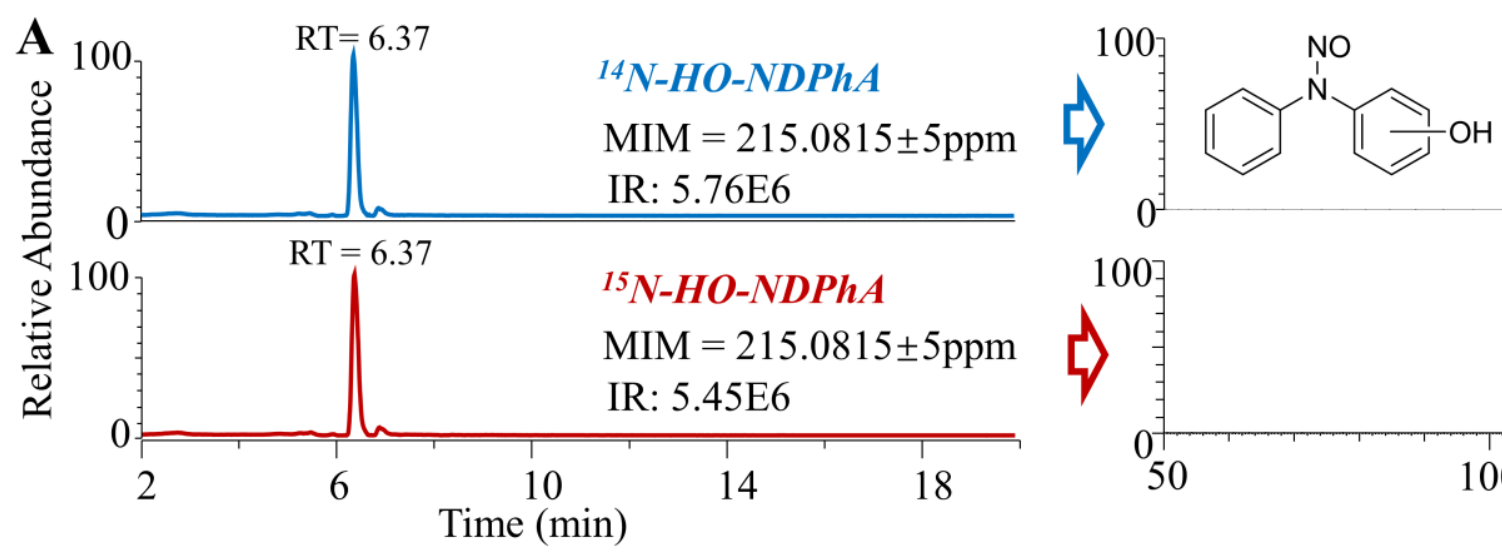

$\left[\mathbf{C}_{\mathbf{1 2}} \mathbf{H}_{\mathbf{1 0}} \mathbf{O}^{\mathbf{1 4}} \mathbf{N}_{\mathbf{2}}\right]^{+} \longrightarrow 198.0789$

$\begin{array}{llll}\mathbf{0 . 6 2} \mathbf{p p m} & 168.0809 & \left.\mathbf{C}_{12} \mathbf{H}_{11} \mathbf{O}_{2}{ }^{14} \mathbf{N}_{2}\right]^{+} & \mathrm{m} / \mathrm{z}=215.0815\end{array}$

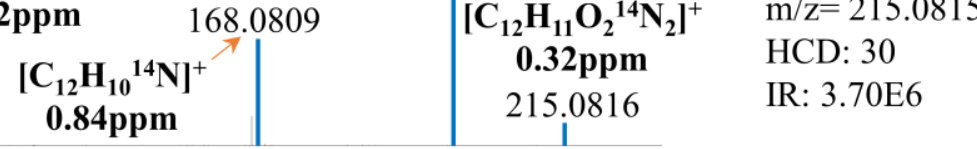

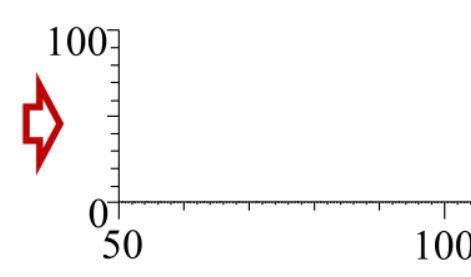

$\left[\mathrm{C}_{12} \mathrm{H}_{10} \mathrm{O}^{14} \mathrm{~N}^{15} \mathrm{~N}\right]^{+}$ $\rightarrow 199.0755$

0.88ppm 168.0810

$\left[\begin{array}{c}{\left[\mathbf{C}_{\mathbf{1 2}} \mathbf{H}_{\mathbf{1 1}} \mathbf{O}_{\mathbf{2}}{ }^{14} \mathbf{N}^{15} \mathbf{N}\right]} \\ \mathbf{0 . 1 4} \mathbf{p p m} \\ 216.0781 \\ \end{array}\right.$

RT: $6.38 \mathrm{~min}$

$\mathrm{m} / \mathrm{z}=216.0785$

HCD: 30

IR: $3.96 \mathrm{E} 6$

\section{B \\ B}

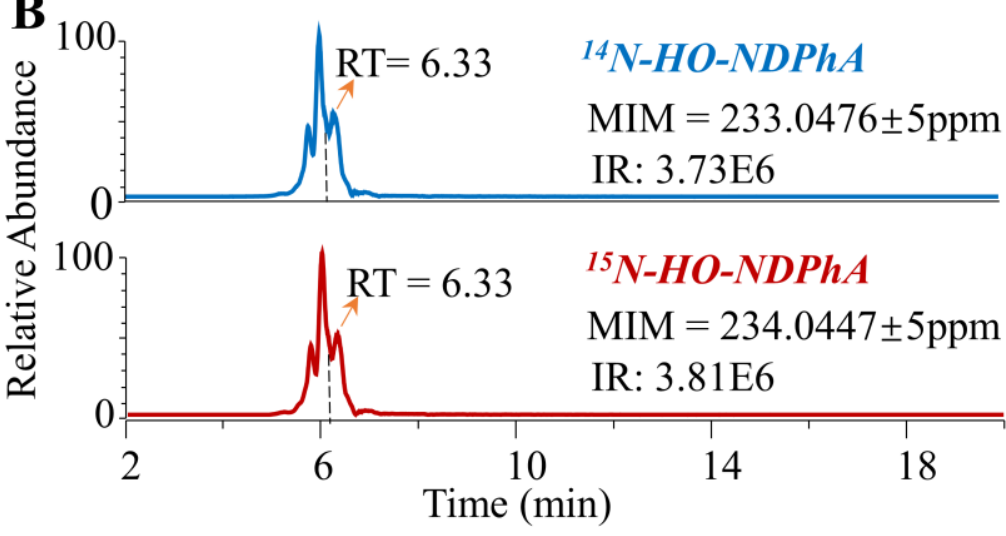

Figure S6. The same retention behaviors and fragmentation patterns of ${ }^{14} \mathrm{~N} /{ }^{15} \mathrm{~N}$-ions were shown in monoisotopic mass (MIM) chromatograms and MS ${ }^{2}$ spectra of HO-NDPhA and Cl-NDPhA produced in chloramination of DPA, respectively. $(A)$ Neutral losses of OH and NO in $\mathrm{MS}^{2}$ spectra of ${ }^{14} \mathrm{~N} /{ }^{15} \mathrm{~N}-\mathrm{HO}-\mathrm{NDPhA}$. (B) Neutral losses of $\mathrm{Cl}$ and $\mathrm{NO}$ in $\mathrm{MS}^{2}$ spectra of ${ }^{14} \mathrm{~N} /{ }^{15} \mathrm{~N}-\mathrm{Cl}-\mathrm{NDPhA}$. 
A

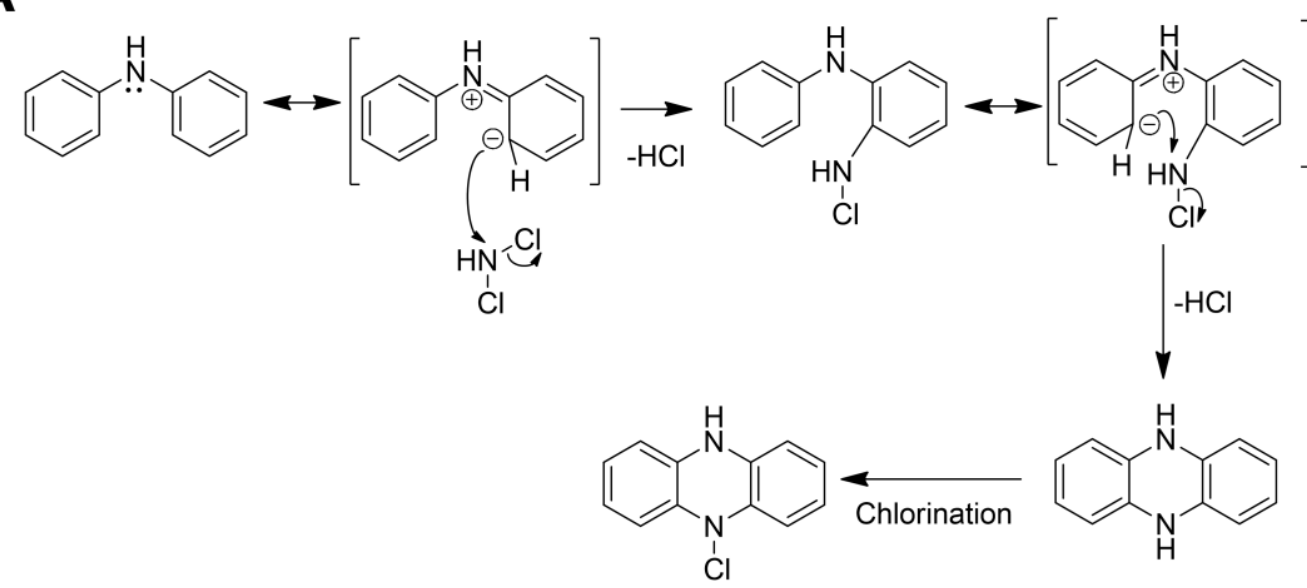

B

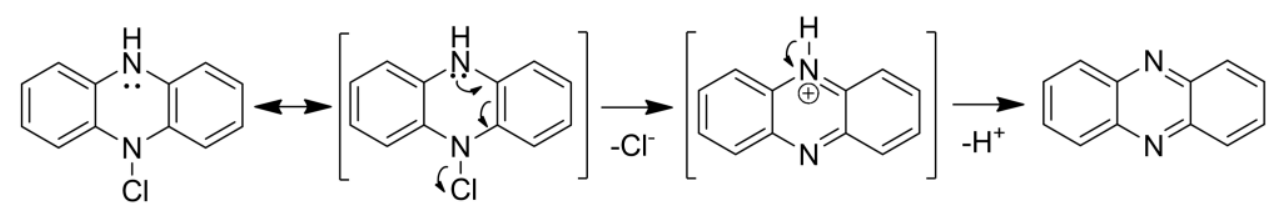

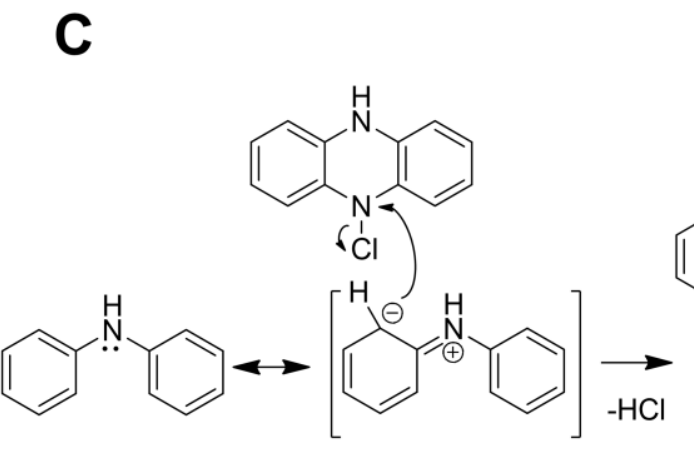<smiles>c1ccc(Nc2ccccc2N2c3ccccc3Nc3ccccc32)cc1</smiles><smiles>c1ccc(N2c3ccccc3Nc3ccccc32)cc1</smiles>

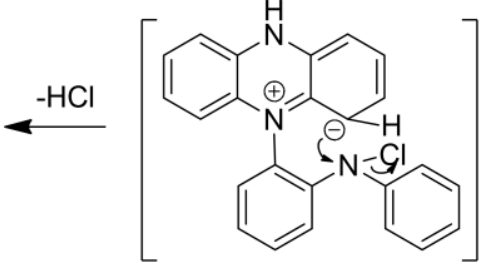

Figure S7. Proposed formation pathway of Cl-DiH-Phe (A), Phe (B) and Cyc-DiDPA (C) from the reaction of DPA with dichloramine. (A) A carbanion resonance structure of DPA successively reacted with dichloramine to form instantaneous intermediate 5,10-dihydro-phenazine (DiH-Phe), and then $\mathrm{DiH}-\mathrm{Phe}$ was rapidly chlorinated by chloramines to form $\mathrm{Cl}$-DiH-Phe. (B) By elimination reaction of intramolecular resonance to lose the $\mathrm{N}-\mathrm{Cl}$ bond, Cl-DiH-Phe could further produce Phe. (C) By attacking $\mathrm{N}-\mathrm{Cl}$ with the carbanion in a resonance structure to lose a $\mathrm{HCl}, \mathrm{Cl}-\mathrm{DiDPA}$ with $\mathrm{N}-\mathrm{Cl}$ could further form Cyc-DiDPA. 


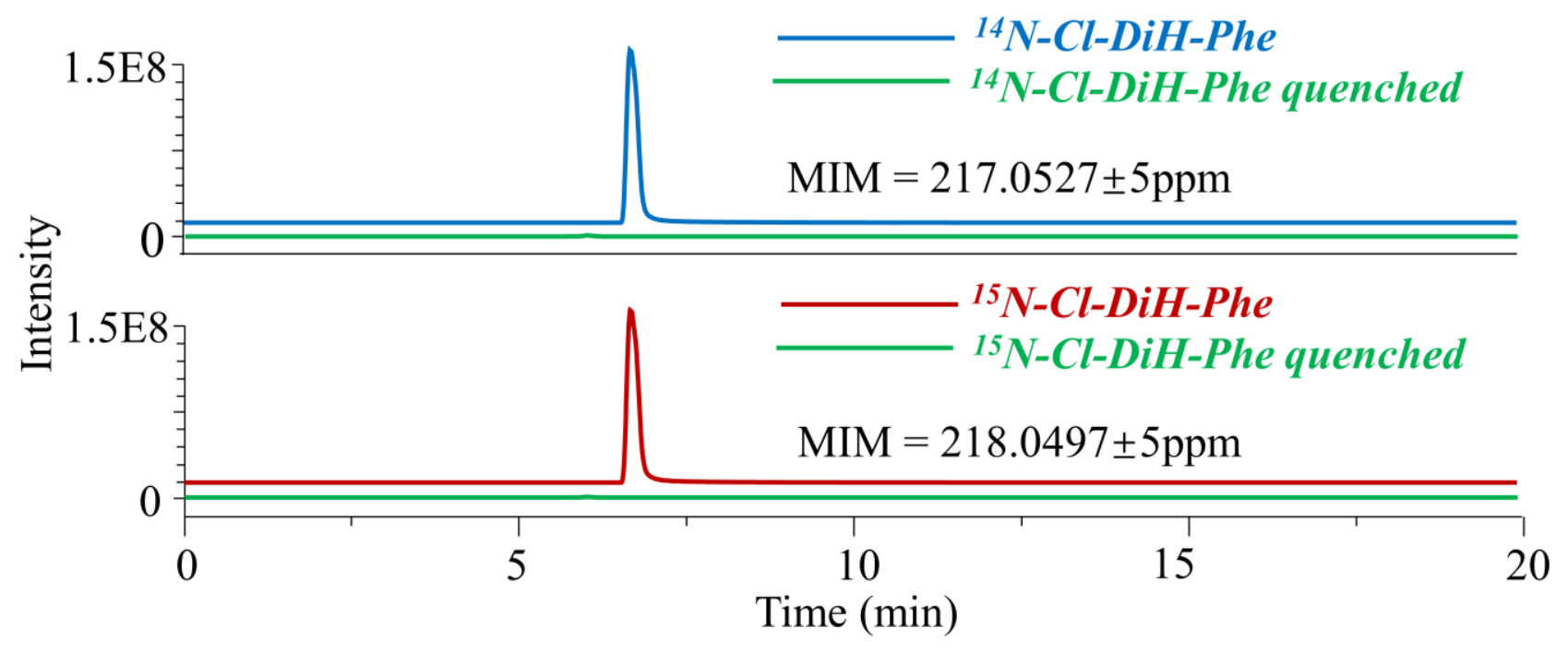

Figure S8. After quenching by $2 \mathrm{mM}$ ascorbic acid (green), ${ }^{14} \mathrm{~N} /{ }^{15} \mathrm{~N}-\mathrm{Cl}-\mathrm{DiH}-\mathrm{Phe}$ (blue and red) disappeared as shown in monoisotopic mass (MIM) chromatograms, suggesting existence of the $\mathrm{N}-\mathrm{Cl}$ bond. The reaction conditions were chloramines/DPA of $100 / 1$ at $\mathrm{pH} 7$ for $12 \mathrm{~h}$. 


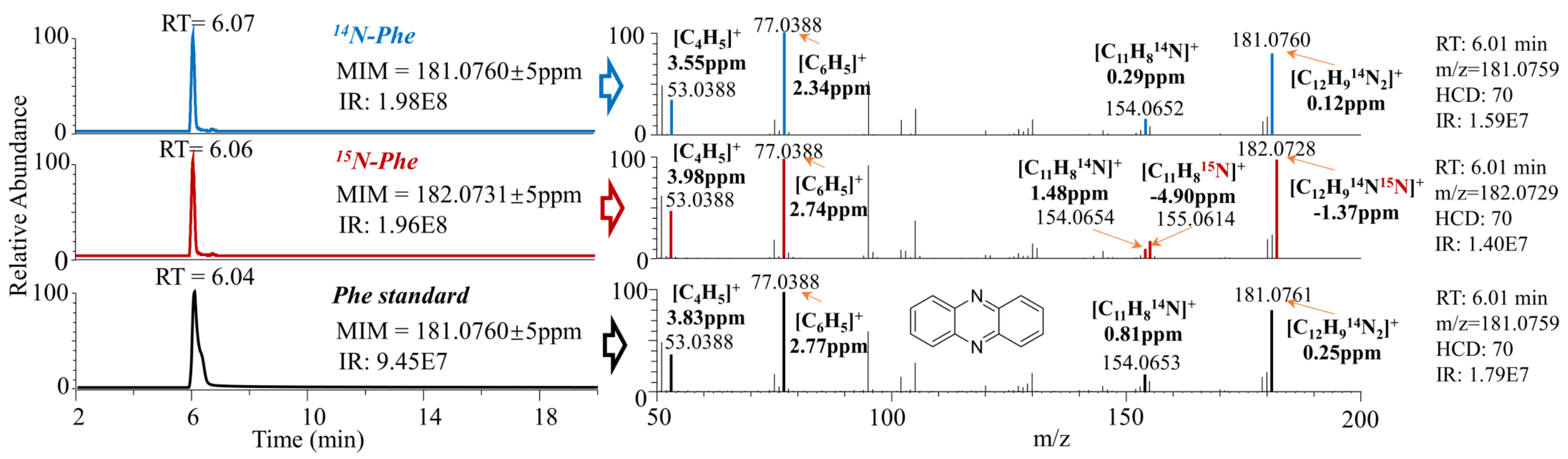

Figure S9. The same retention behaviors and fragmentation patterns of ${ }^{14} \mathrm{~N} /{ }^{15} \mathrm{~N}-\mathrm{Phe}$ (Blue and Red) produced in chloramination and Phe standard (Black) shown in monoisotopic mass (MIM) chromatograms and $\mathrm{MS}^{2}$ spectra, respectively. A pair of fragment ions $\left(\left[\mathrm{C}_{11} \mathrm{H}_{8}{ }^{14} \mathrm{~N}\right]^{+}\right.$and $\left.\left[\mathrm{C}_{11} \mathrm{H}_{8}{ }^{15} \mathrm{~N}\right]^{+}\right)$with similar intensity were shown in $\mathrm{MS}^{2}$ spectrum of ${ }^{15} \mathrm{~N}$-Phe, indicating ${ }^{14} \mathrm{~N}$ (originally from the DPA molecule) and ${ }^{15} \mathrm{~N}$ atoms (from chloramines) were in the enantiomeric position in ${ }^{15} \mathrm{~N}-\mathrm{Phe}$. 


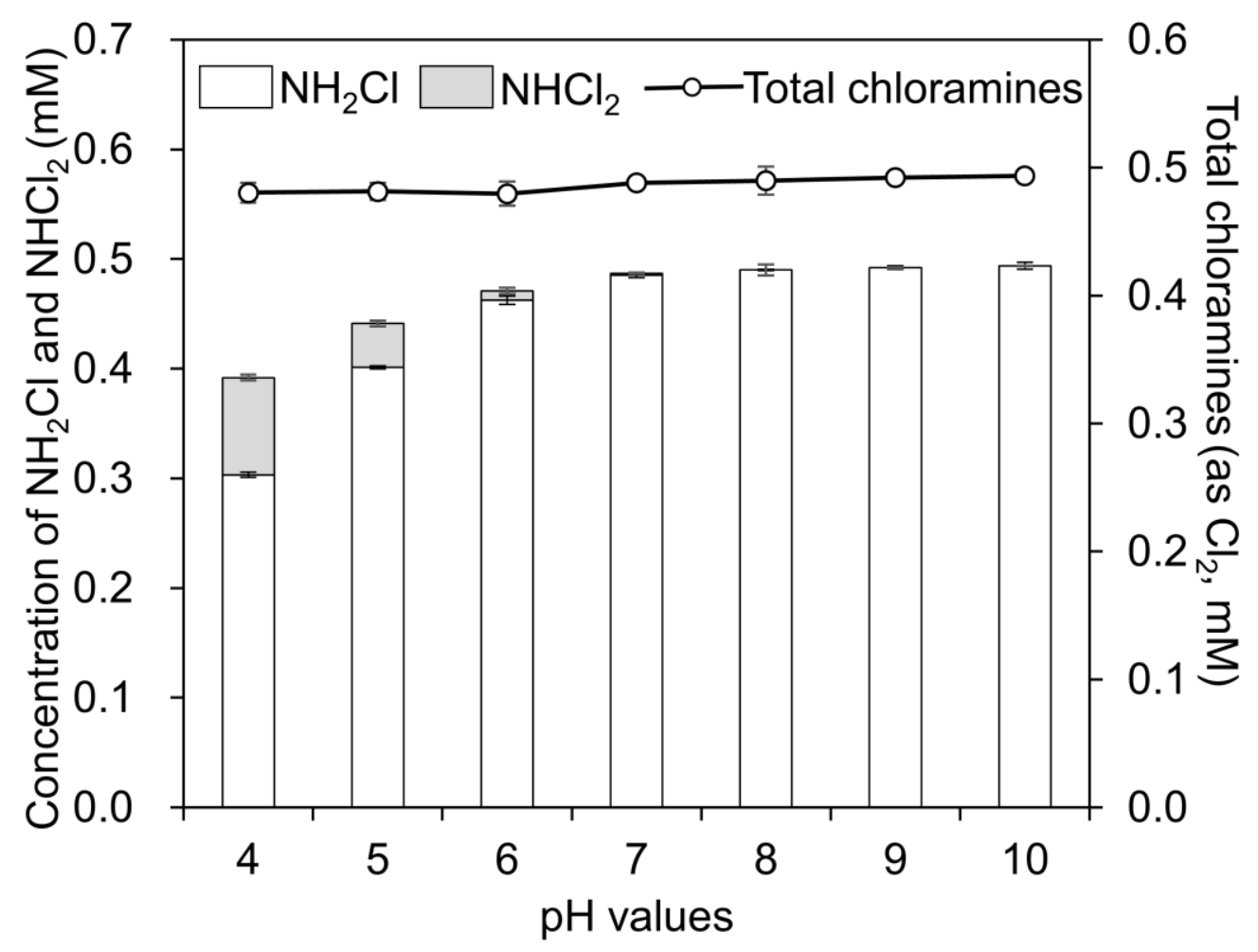

Figure S10. Concentrations of monochloramine and dichloramine in the $0.5 \mathrm{mM}$ solutions of chloramines (as $\left.\mathrm{Cl}_{2}\right)$ at $\mathrm{pH} 4-10$. 


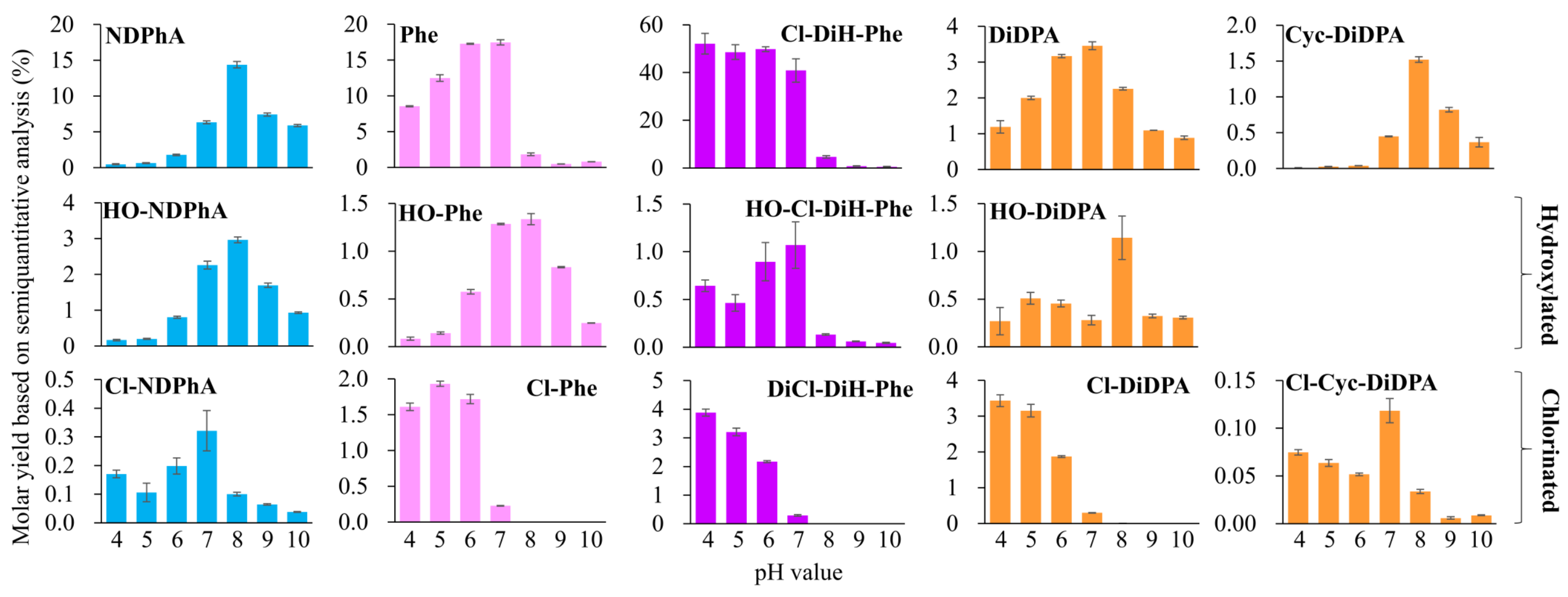

Figure S11. Effect of $\mathrm{pH}$ on formation of the four groups of N-DBPs and hydroxylated/chlorinated products. Maximum molar yields of all chlorinated products were observed at $\mathrm{pH} \leqslant 7$. Except for $\mathrm{HO}-\mathrm{Cl}-\mathrm{DiH}-\mathrm{Phe}$, maximum formation yields of hydroxylated products were found at $\mathrm{pH} \geqslant 7$. 


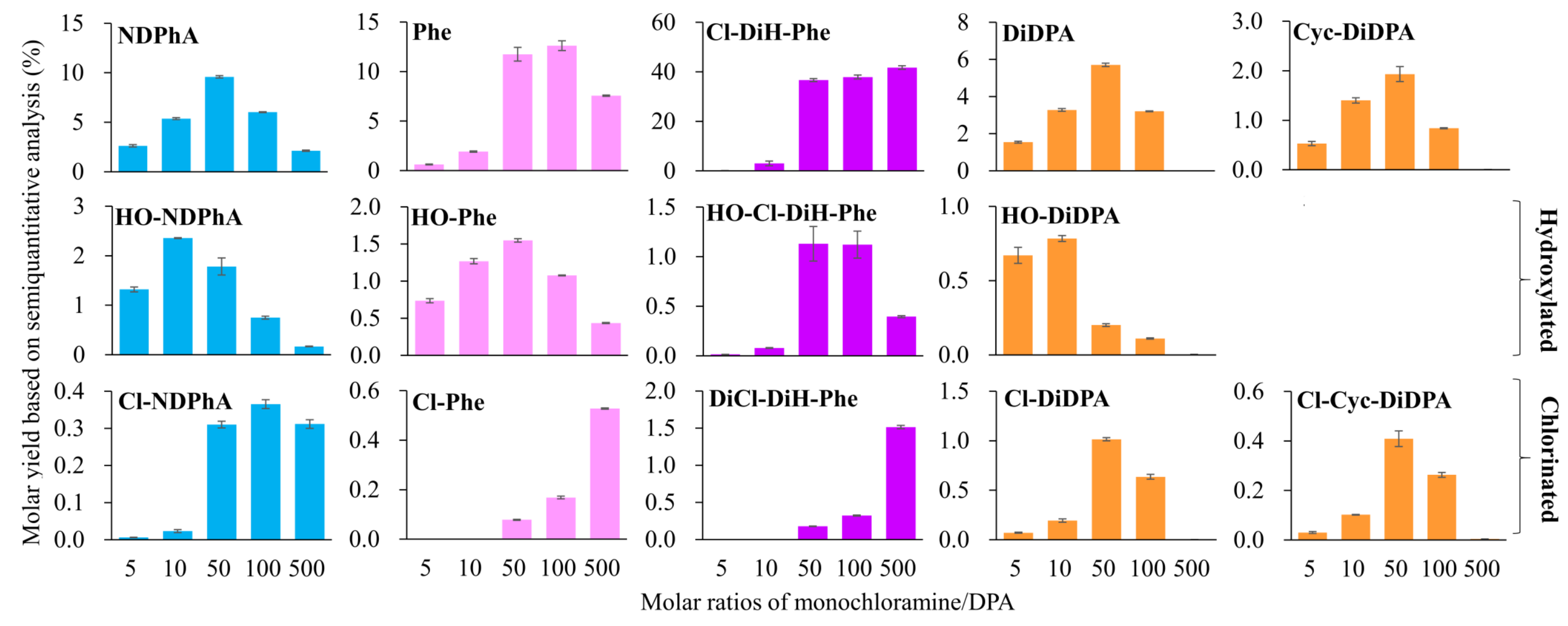

Figure S12. Effect of chloramine doses on formation of the four groups of N-DBPs and hydroxylated/chlorinated products, in which maximum molar yields of hydroxylated products were found with molar ratios of chloramines/DPA at $10-100$, and dependences of chlorinated DBPs on disinfectants doses were noticed. 


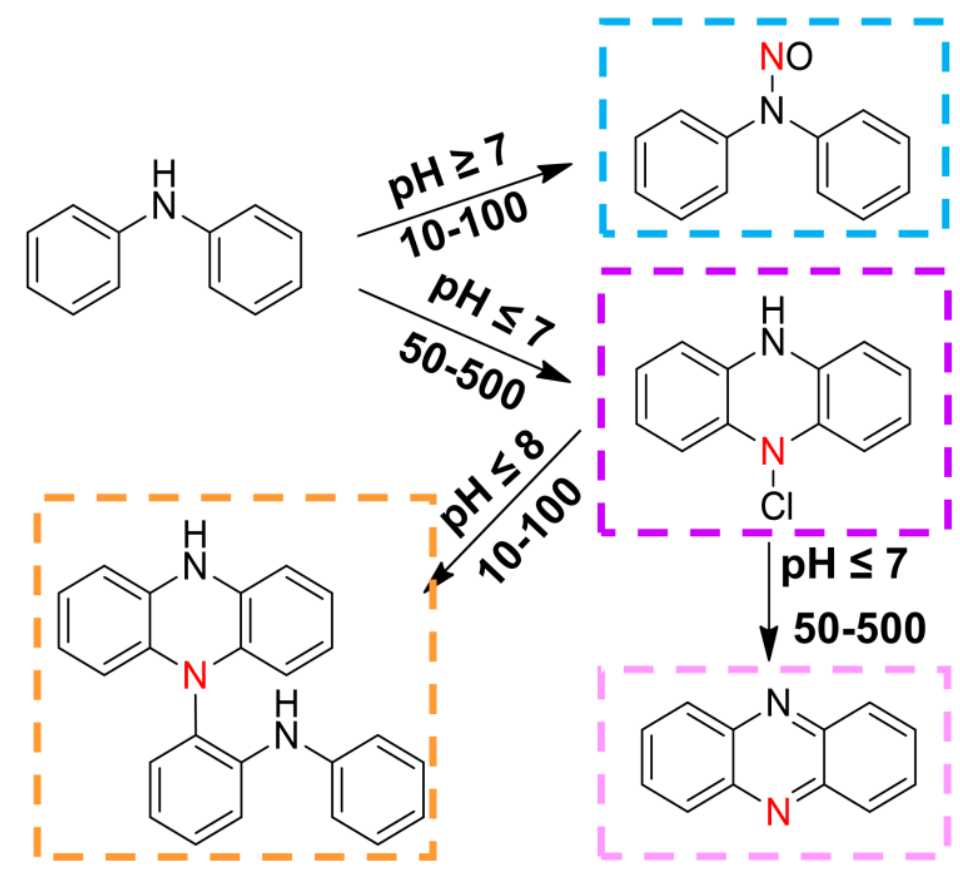

Figure S13. Main reaction conditions for preferred formation of the four groups of N-DBPs from DPA. 

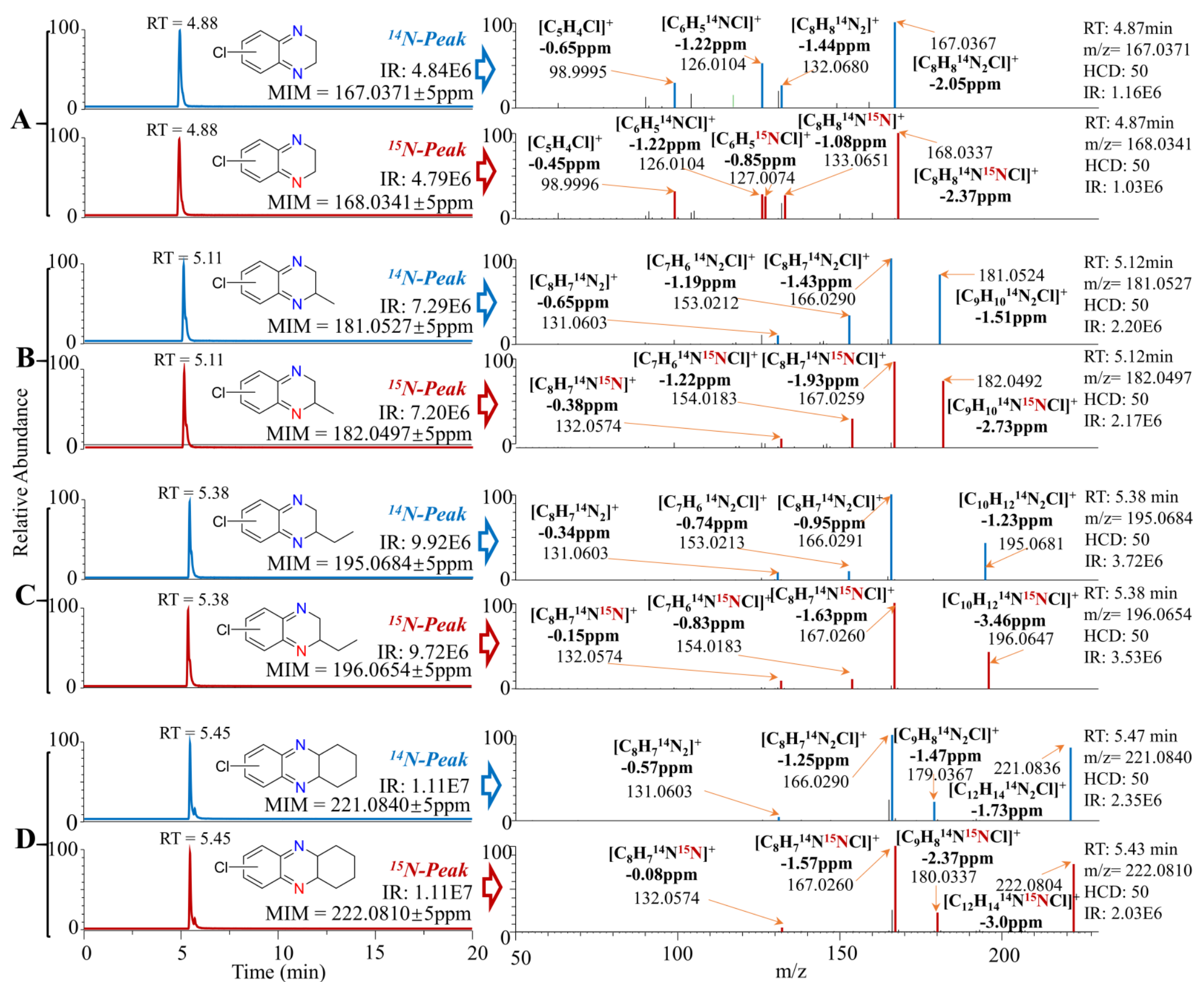

Figure S14. Increased retention of monochlorinated cyclized quinonediimines with growth of alkyl chain lengths was shown in the monoisotopic mass (MIM) chromatograms. Fragment ions $\left[\mathrm{C}_{8} \mathrm{H}_{7}{ }^{14} \mathrm{~N}_{2} \mathrm{Cl}\right]^{+}$and $\left[\mathrm{C}_{8} \mathrm{H}_{7}{ }^{14} \mathrm{~N}^{15} \mathrm{NCl}\right]^{+}$with the maximum abundance as shown in $\mathrm{MS}^{2}$ spectra of ${ }^{14} \mathrm{~N}$ and ${ }^{15} \mathrm{~N}$ peaks were cyclized molecular skeleton. Monochlorinated cyclized quinonediimines were produced in chloramination of N-ethylaniline (A), N-propylaniline (B), N-butylaniline (C), and N-cyclohexylaniline (D). 


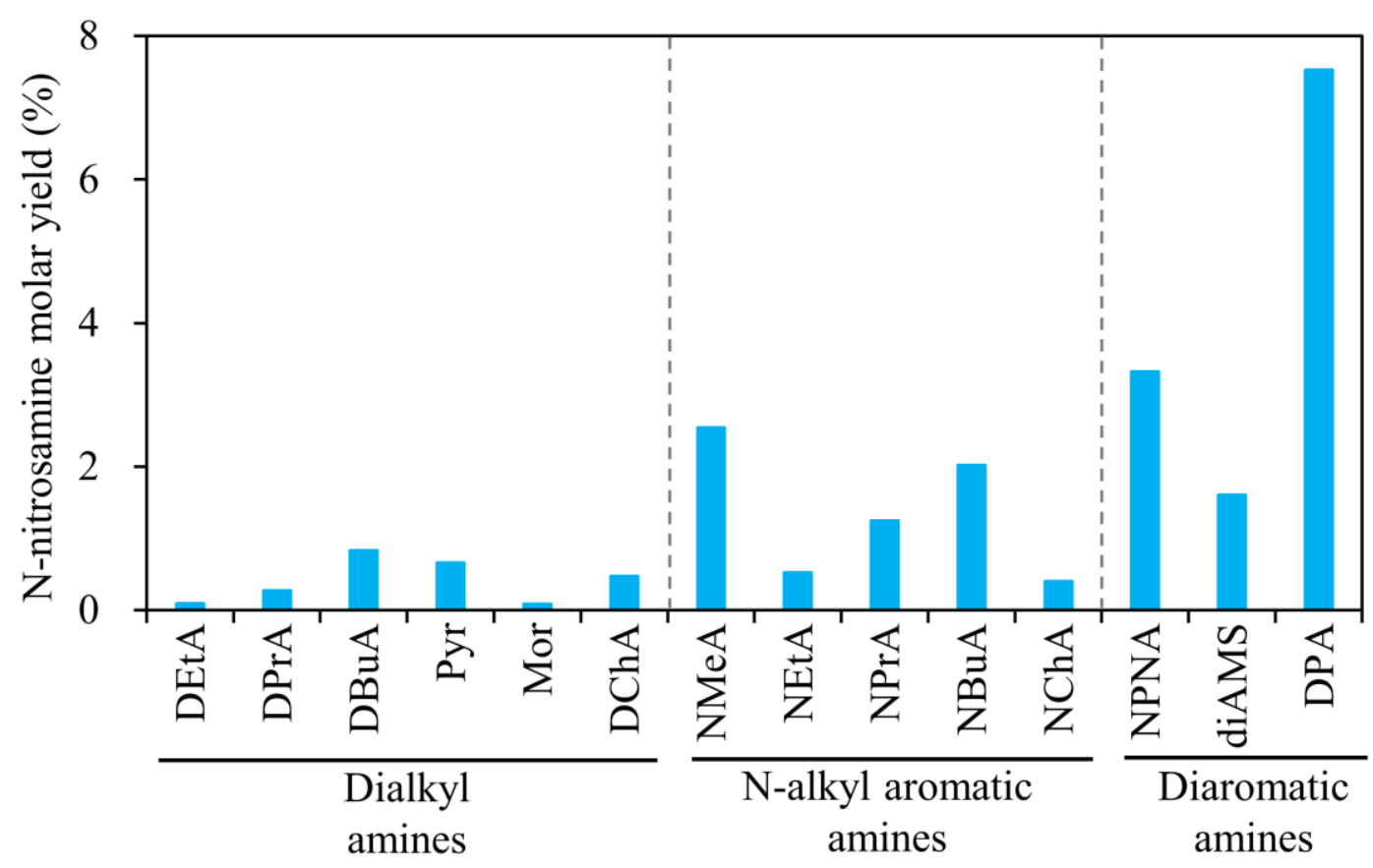

Figure S15. Molar yields of N-nitrosamines from different secondary amine precursors with chloramines/precursor molar ratios of $100 / 1$ at $\mathrm{pH} 7$ after $12 \mathrm{~h}$ treatment. Higher molar yields from $\mathrm{N}$-alkyl aromatic and diaromatic amines were observed compared with those from dialkyl amines. 


\section{N-nitroso diaromatic amine}
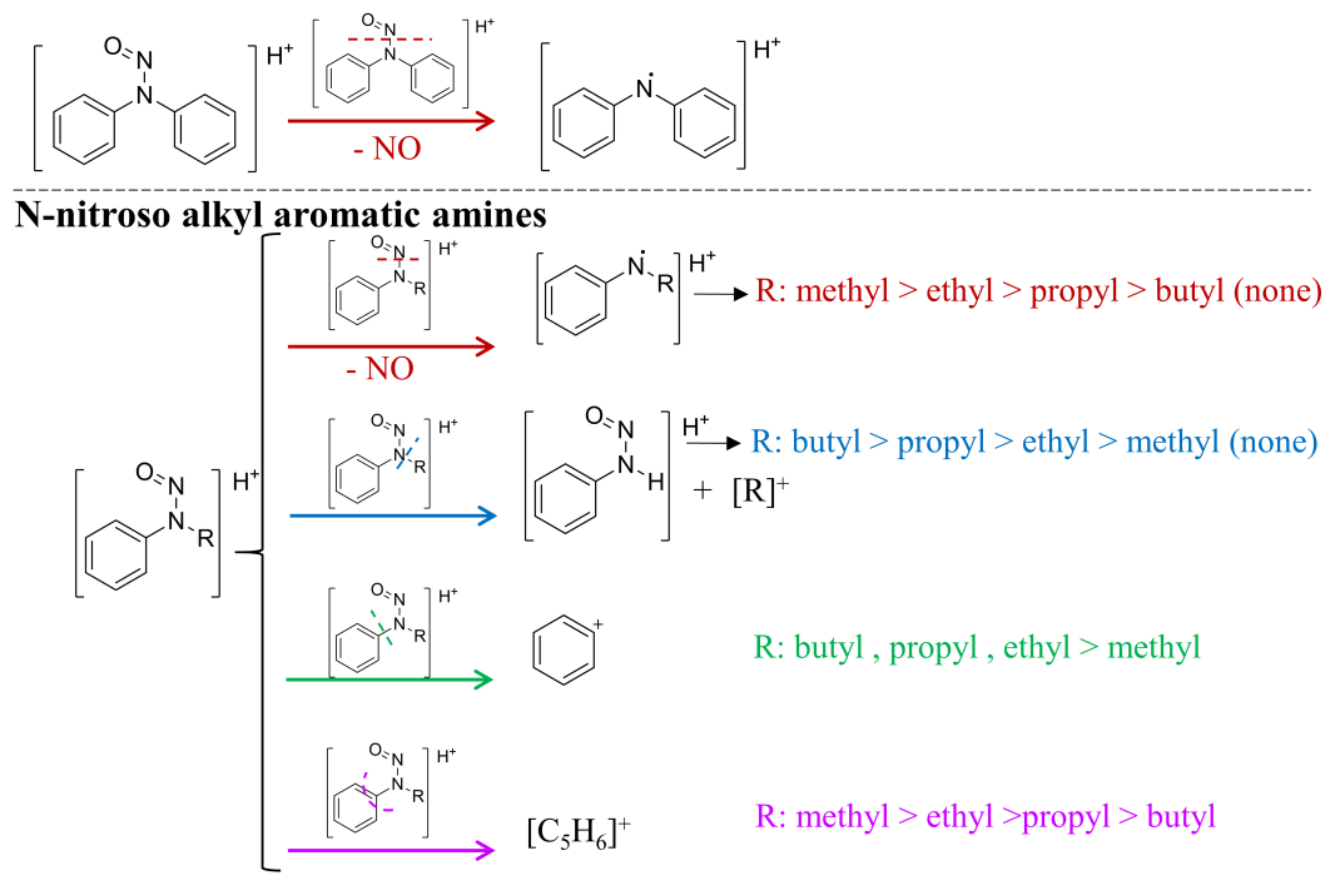

$$
\begin{aligned}
& \mathrm{N} \text {-nitroso dialkyl amine }
\end{aligned}
$$

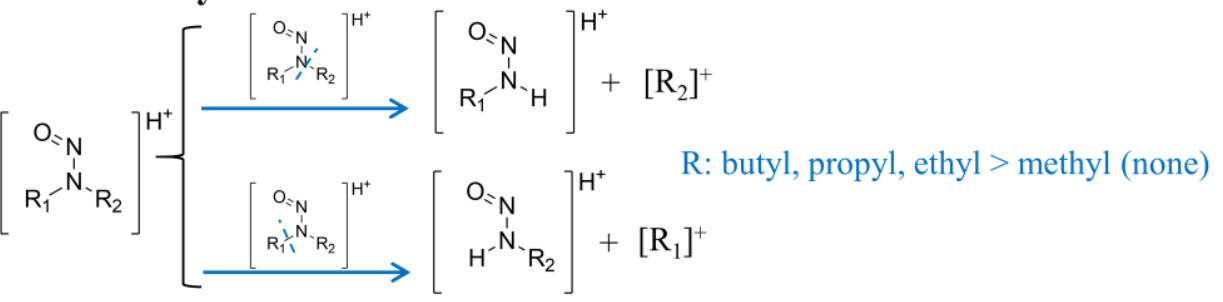

Figure S16. Main fragmentation patterns of different $\mathrm{N}$-nitrosoamines in $\mathrm{MS}^{2}$ spectra acquired by HRMS at $30 \% \mathrm{HCD}$ energy. Diagnostic loss of NO was observed in $\mathrm{MS}^{2}$ spectra of $\mathrm{N}$-nitroso diaromatic amines, but loss of alkyl moieties was specific for $\mathrm{N}$ nitroso dialkyl amines. Losses of both the NO and alkyl moieties appeared in $\mathrm{MS}^{2}$ spectra of N-nitroso alkyl aromatic amines, which were related to the length of alkyl moieties. It should be noted that standards of N-nitrosopropylaniline and $\mathrm{N}$ nitrosobutylaniline were not commercially available, and the $\mathrm{MS}^{2}$ spectra were obtained from the chloramine-treated samples of corresponding amine precursors. 